\title{
Fungi and lichens recorded during the Cryptogam Symposium on Natural Beech Forests, Slovakia 2011
}

\author{
Slavomír AdAMČíK ${ }^{1} *$, ERIK AUdE ${ }^{2}$, Claus BÄSSler ${ }^{3}$, MORTEN CHRISTENSEN $^{4}$, \\ KLAAS VAN DORT ${ }^{5}$, ÖRJAN FRITZ ${ }^{6}$, STANISLAV GLEJDURA ${ }^{7}$, \\ JaCOB Heilmann-Clausen ${ }^{8}$, JAN HoleC ${ }^{9}$, SOŇA JanČOviČOvá ${ }^{10}$, Vladimír KunCA ${ }^{7}$, \\ ANNA LACKOVIČOVÁ ${ }^{1}$, MiCHAEL LÜTH ${ }^{11}$, PÉTER ÓDOR ${ }^{12}$ \\ ${ }^{1}$ Slovak Academy of Sciences, Institute of Botany, Dúbravská cesta 9, SK-845 23 Bratislava, \\ Slovakia; slavomir.adamcik@savba.sk \\ ${ }^{2}$ Rørvangen 2B, DK-8520 Lystrup, Denmark \\ ${ }^{3}$ Bavarian Forest National Park, Freyunger Str. 2, DE-94481 Grafenau, Germany \\ ${ }^{4}$ Grontmij, Granskoven 8, DK-2600 Glostrup, Denmark \\ ${ }^{5}$ Forestfun, Leeuweriksweide 186, NL-6708 Wageningen, the Netherlands \\ ${ }^{6}$ Lingonstigen 6, SE-296 34 Åhus, Sweden \\ ${ }^{7}$ Technical University in Zvolen, Faculty of Ecology and Environmental Sciences, T. G. Masaryka 24, \\ SK-960 53 Zvolen, Slovakia \\ ${ }^{8}$ Centre for Macroecology, Evolution and Climate, Natural History Museum of Denmark, University \\ of Copenhagen, DK-2100 København, Denmark \\ ${ }^{9}$ National Museum, Mycological Department, Cirkusová 1740, CZ-193 00 Praha 9, Czech Republic \\ ${ }^{10}$ Comenius University in Bratislava, Faculty of Natural Sciences, Department of Botany, Révová 39, \\ SK-811 02 Bratislava, Slovakia \\ ${ }^{11}$ Büro für Umweltplanung, Emmendinger Str. 32, DE-79106 Freiburg, Germany \\ ${ }^{12}$ MTA Centre for Ecological Research, Institute of Ecology and Botany, Alkotmány u. 2-4, \\ HU-2163 Vácrátót, Hungary \\ *corresponding author
}

Adamčík S., Aude E., Bässler C., Christensen M., Dort K. van, Fritz Ö., Glejdura S., Heilmann-Clausen J., Holec J., Jančovičová S., Kunca V., Lackovičová A., Lüth M., Ódor P. (2016): Fungi and lichens recorded during the Cryptogam Symposium on Natural Beech Forests, Slovakia 2011. - Czech Mycol. 68(1): 1-40.

In September 2011, an international team of cryptogam experts visited seven national nature reserves in five mountain areas of Slovakia: Havešová and Stužica in the Poloniny Mts., Vihorlat in the Vihorlatské vrchy Mts., Oblík in the Slanské vrchy Mts., Dobročský prales and Klenovský Vepor in the Veporské vrchy Mts. and Badínsky prales in the Kremnické vrchy Mts. The reserves were selected to represent examples of the best protected old-growth beech forests in the country. The aim was to study the diversity of wood-inhabiting fungi on fallen beech logs and epiphytic lichens on standing beech trees. In total, 215 fungal species and 128 lichens were recorded on beech wood and bark, and 27 fungi and 26 lichens on additional substrates. The site of the highest conservation value is Stužica with 126 fungi and 79 lichens recorded on beech, of which 12 fungi and 19 lichens are indicators of high nature conservation value. Combined with historical records, a total of 19 non-lichenised fungal indicators are now reported from the site, making it the highest ranked natural beech forest in Europe. The second most important reserve for fungal diversity is Havešová with 121 species, including 14 indicator species recorded on beech wood. For lichens, the second most important reserve is Klenovský Vepor with 69 species including 18 lichen indicators recorded on beech. Nine fungus 
species are here reported as new to Slovakia: Asterostroma medium, Entoloma hispidulum, E. pseudoparasiticum, Gloeohypochnicium analogum, Hohenbuehelia valesiaca, Hymenochaete ulmicola, Hypocrea parmastoi, Melanomma spiniferum and Scutellinia colensoi. Lichen species Alyxoria ochrocheila is reported as new to Slovakia and Lecanographa amylacea, which was considered extinct in the Slovak Red list, was also recorded. This is the first list of wood-inhabiting fungi and epiphytic lichens of old-growth beech forests in Slovakia, and hence an important contribution to the exploration of biodiversity in Slovakia.

Key words: old-growth beech forests, diversity, conservation, indicator, red-list, Europe.

Article history: received 27 July 2015, revised 11 December 2015, accepted 21 December 2015, published online 1 February 2016.

Adamčík S., Aude E., Bässler C., Christensen M., Dort K. van, Fritz Ö., Glejdura S., Heilmann-Clausen J., Holec J., Jančovičová S., Kunca V., Lackovičová A., Lüth M., Ódor P. (2016): Huby a lišajníky zaznamenané počas podujatia Cryptogam Symposium on Natural Beech Forests in Slovakia, 2011. - Czech Mycol. 68(1): 1-40.

V septembri roku 2011 navštívil medzinárodný tím kryptogamológov sedem národných prírodných rezervácii v piatich pohoriach Slovenska: Havešová a Stužica v Poloninách, Vihorlat vo Vihorlatských vrchoch, Oblík v Slanských vrchoch, Dobročský prales a Klenovský Vepor vo Veporských vrchoch a Badínsky prales v Kremnických vrchoch. Rezervácie boli vybrané tak, aby predstavovali čo najzachovanejšie bukové lesy pralesovitého charakteru. Cielom bolo štúdium diverzity húb rastúcich na ležiacich kmeňoch buka a epifytických lišajníkov na stojacich kmeňoch buka. Celkovo bolo zistených 215 druhov húb a 128 lišajníkov na dreve a borke bukov a 27 druhov húb a 26 lišajníkov na iných substrátoch. Najhodnotnejšou rezerváciou je Stužica so 126 druhmi húb a 79 druhmi lišajníkov zaznamenaných na buku, z ktorých 12 druhov húb a 19 lišajníkov sú indikátory vysokej prírodnej hodnoty. Spolu s historickými údajmi je v súčasnosti z tejto lokality známych 19 indikačných druhov nelichenizovaných húb, čo ju umiestňuje na popredné miesto v Európe. Druhou najvýznamnejšou rezerváciou z hladiska druhovej bohatosti húb je Havešová so 121 druhmi vrátane 14 indikačných druhov zaznamenaných na buku. Pre lišajníky je druhou v poradí rezervácia Klenovský Vepor so 69 druhmi vrátane 18 indikátorov zaznamenaných na buku. V rámci výskumu sa podarilo zistit devät druhov húb, ktoré dosial' neboli zo Slovenska známe: Asterostroma medium, Entoloma hispidulum, E. pseudoparasiticum, Gloeohypochnicium analogum, Hohenbuehelia valesiaca, Hymenochaete ulmicola, Hypocrea parmastoi, Melanomma spiniferum a Scutellinia colensoi. Z lišajníkov je prvým nálezom pre Slovensko Alyxoria ochrocheila. Potvrdený bol aj druh Lecanographa amylacea, ktorý je v červenom zozname lišajníkov Slovenska uvedený ako vyhynutý. Príspevok predstavuje prvý ucelený zoznam húb a lišajníkov rastúcich na bukoch v siedmich pralesovitých bučinách Slovenska a významnou mierou prispieva k poznaniu biodiverzity tejto krajiny.

\section{INTRODUCTION}

Slovakia is situated in the western part of the Carpathian Mts., and mountain chains dominate major parts of the country. The Poloniny National Park (Eastern Carpathians, Slovakia) is known to have some of the most well-preserved natural beech forest reserves in Europe, several of which are included in the list of World Heritage sites (Anonymus 1, on-line). Most authors of this contribution are mem- 
bers of an informal group of cryptogam experts of beech forests, nicknamed the 'Beech Boys'. It is our aim to compare species diversity, community composition and conservation value of beech forests across Europe with a special focus on cryptogams associated with dead wood and veteran trees. The aim of our longterm research is to develop a system for assessing the nature conservation value of beech forests based on indicator species. A preliminary system for assessing the conservation value of beech forests based only on wood-inhabiting fungi was presented by Christensen et al. (2004). According to this system, the Poloniny National Park includes the most valuable beech forest reserves in Europe. As yet, no other areas in Slovakia had been evaluated following this system, and for many potentially important reserves the knowledge of our focal species groups is very low. For this reason, Slovakia was a logical destination for the Beech Boys in 2011. This event, officially called 'Cryptogam Symposium on Natural Beech Forests in Slovakia' took place from 24 September to 1 October 2011 and was prepared in cooperation with staff members of protected areas and the Slovak Environmental Agency. In addition to Stužica National Nature Reserve (NNR) and Havešová NNR in the Poloniny Mts., we visited Vihorlat NNR in the Vihorlatské vrchy Mts., Oblík NNR in the Slanské vrchy Mts., Dobročský prales NNR and Klenovský Vepor NNR in the Veporské vrchy Mts. and Badínsky prales NNR in the Kremnické vrchy Mts.

Wood-inhabiting fungi in Slovakia. The Poloniny Mts. is the best known area concerning fungal diversity in Slovakia: a total of 1199 taxa of macrofungi were known from the area before the present investigation (Adamčík et al. 2003, 2007). Previous investigations of wood-inhabiting fungi resulted in the highest score of natural beech forest indicators (in sense of Christensen et al. 2004) known in Europe for Rožok National Nature Reserve (NNR) and Stužica NNR in the Poloniny National Park (Kuthan et al. 1999, Adamčík et al. 2007). For the other areas investigated during our symposium, several data on occurrence of wood-inhabiting fungi were published in monographic studies on polypores (Kotlaba 1984), corticioid fungi (Hagara 2001, 2004) and tomentelloid fungi (Svrček 1960). More comprehensive reports on wood-inhabiting fungi have also been published for Dobročský prales NNR (Kotlaba \& Pouzar 1962, Varjú 1994), while in contrast the diversity of wood-inhabiting fungi in the Kremnické vrchy Mts., Slanské vrchy Mts. and Vihorlatské vrchy Mts. is poorly known from fungistic studies (e.g. Kult 1991, Mihál 1996, Ripková et al. 2007).

Epiphytic lichens in Slovakia. Lichens are a relatively well-investigated group of cryptogams in Slovakia, compared to non-lichenised fungi. More than a thousand Slovak lichenological studies deal with the occurrence and distribution of lichens on various substrates, their bio-indication value, conservation, and changes of their diversity in time and space. According to these studies, 1628 lichen species are known from the country. A considerable number of re- 
ports focus on epiphytic lichens, but none of them deal exclusively with beech species. Lichens associated with living trees in Slovakia were the subject of largescale mapping in the period 1970 to 1981 . Less than 200 species were recorded during this mapping period, but more than 400 epiphytic lichens had been recorded in Slovakia before (Pišút 1999), the highest number being from the Tatra Mts. (327 species reported by Lisická 2006). The most important publications dealing with the areas visited during our studies concern species in (semi-)natural forest ecosystems in the Bukovské vrchy Mts. (the major part of Poloniny National Park) (Pišút et al. 2007, Guttová 1997), Dobročský prales National Nature Reserve (Lackovičová \& Pišút 2004) and Stužica National Nature Reserve (Pišút \& Lackovičová 1992, Pišút et al. 2007, Vondrák et al. 2015).

\section{MATERIAL AND METHODS}

Investigated sites. From 25 to 30 September 2011, we visited selected National Nature Reserves (NNR) in Slovakia as listed below (Fig. 1). Geographical coordinates match approximately the central part of the reserves.

25 Sep 2011 - Havešová NNR: Poloniny Mts., virgin beech forest (mixed with Fraxinus excelsior and Acer spp. in upper parts), alt. 440-740 m, coord. 49 $09^{\prime} 40^{\prime \prime} \mathrm{N}, 22^{\circ} 20^{\prime} 00^{\prime \prime} \mathrm{E}$, area $1.71 \mathrm{~km}^{2}$, exposition S, SE and SW, Central European mapping grid quadrate Q 6999d (Niklfeld 1971).

26 Sep 2011 - Stužica NNR: Poloniny Mts., virgin forest, dominated by Fagus sylvatica and Abies $a l b a$, in upper parts also by Acer pseudoplatanus, alt. $650-1200 \mathrm{~m}$, coord. of the central part $49^{\circ} 04^{\prime} 40^{\prime \prime}$ $\mathrm{N}, 22^{\circ} 32^{\prime} 30^{\prime \prime} \mathrm{E}$, area $7.6 \mathrm{~km}^{2}$, slopes and terraces above the Stužická rieka valley (river flowing in E direction), Q 69'01a.

27 Sep 2011 - Vihorlat NNR: Vihorlatské vrchy Mts., deciduous virgin forest dominated by Fagus sylvatica with frequent Acer pseudoplatanus, A. platanoides and Fraxinus excelsior trees, alt. $800-1075 \mathrm{~m}$, coord. $48^{\circ} 533^{\prime} 31^{\prime \prime} \mathrm{N}, 22^{\circ} 06^{\prime} 56^{\prime \prime} \mathrm{E}$, area $0.29 \mathrm{~km}^{2}$, surrounding of the highest peak in the Vihorlatské vrchy Mts. with steep, mostly N-facing slopes, Q 7198a.

28 Sep 2011 - Oblík NNR: Slanské vrchy Mts., deciduous virgin forest dominated by Fagus sylvatica, alt. 595-925 m, coord. 48 $58^{\prime} 24^{\prime \prime} \mathrm{N}, 21^{\circ} 28^{\prime} 22^{\prime \prime} \mathrm{E}$, area $0.89 \mathrm{~km}^{2}$, almost symmetric, obtuse, conical, isolated volcano hill with rather steep slopes of all expositions, Q 7094b.

29 Sep 2011 - Dobročský prales NNR: Veporské vrchy Mts., mixed virgin forest dominated by Fagus sylvatica and Abies alba, in some parts by Picea abies trees, on ridge with frequent Acer pseudoplatanus, alt. $730-1005 \mathrm{~m}$, coord. $48^{\circ} 41^{\prime} 01^{\prime \prime} \mathrm{N}, 19^{\circ} 40^{\prime} 45^{\prime \prime} \mathrm{E}$, area $0.51 \mathrm{~km}^{2}$, SW slopes above Brôtovo stream, Q 7383b.

29 Sep 2011 - Badínsky prales NNR: Kremnické vrchy Mts., mixed virgin forest with Fagus sylvatica and Abies alba, alt. 690-875 m, coord. 48 41'21" N, 19 $03^{\prime} 15^{\prime \prime} \mathrm{E}$, area $0.3 \mathrm{~km}^{2}$, SE slopes in valley of Badínsky potok stream, Q 7380a.

30 Sep 2011 - Klenovský Vepor NNR: Veporské vrchy Mts., virgin forest represented by various habitats; investigation was conducted in mixed forest of Fagus sylvatica, Picea abies and Abies alba, in upper part of the mountain ridge with Acer pseudoplatanus, alt. 1100-1338 m, coord. $48^{\circ} 41^{\prime} 16^{\prime \prime} \mathrm{N}, 19^{\circ} 45^{\prime} 53^{\prime \prime} \mathrm{E}$, area $1.25 \mathrm{~km}^{2}$, massif of mountain ridge (oriented mostly W-E) represented by various expositions and often rocky surfaces and steep slopes, Q 7384a-b. 


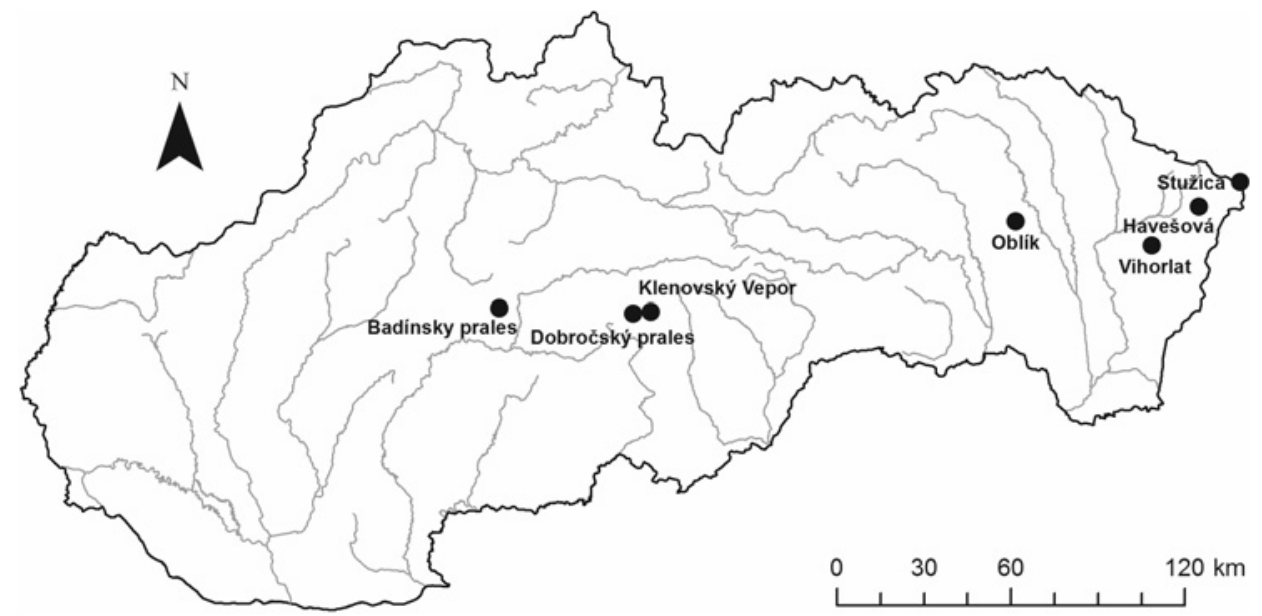

Fig. 1. Geographic position of investigated sites in Slovakia.

Collection and identification of fungi. At each site, beech logs (Fagus sylvatica) were selected by means of stratified sampling with the intention to cover all defined categories in relation to two main criteria: log diameter and stage of decay. Fallen beech logs were categorised according to their diameter at breast height $(1.3 \mathrm{~m})$ into the following classes: (1) $20-49 \mathrm{~cm}$, (2) 50-79 cm, (3) $\mathrm{min} .80 \mathrm{~cm}$. The estimation of decay stages (DS) follows Heilmann-Clausen (2001): DS1 - logs covered with bark without visible signs of decay, DS2 - decay signs indistinct, wood and bark weakly disrupted, DS3 - decay of wood distinct, bark partially loosening or cracking, DS4 - wood strongly damaged but still with visible structure, in major part without bark, DS5 - logs rotten to almost humified. On each selected log, macrofungi were recorded as specified by Ódor et al. (2006). Corticioid basidiomycetes and several ascomycete groups were only sporadically recorded. In total, we studied 30 logs at Havešová, 30 at Stužica, 20 at Vihorlat, 20 at Oblík, 19 at Dobročský prales and 31 at Klenovský Vepor (Tab. 1). Logs of intermediate size and decay stages are overrepresented. This lack of balance in the dataset reflects that some log categories were sparsely represented in the field. Also the limited time available in the field prevented us from finding logs meeting all selection criteria. In addition to the stratified sampling of selected beech logs, additional tree substrates (other beech logs as well as other tree species) were surveyed in order to maximise the number of species recorded at each site. A short visit to Badínsky prales only allowed us to do some opportunistic sampling. 
Tab. 1. Numbers of beech logs investigated for fungal diversity in defined categories of decay and thicknesses. The data originate from all investigated sites with the exception of Badínsky prales NNR. DS - decay stage.

\begin{tabular}{|l|c|c|c|c|c|c|}
\hline \multirow{2}{*}{ Log thickness } & \multicolumn{7}{|c|}{ Number of investigated beech logs per decay stage } \\
\cline { 2 - 7 } & DS1 & DS2 & DS3 & DS4* & DS5 & Total \\
\hline $\mathbf{2 0 - 4 9} \mathbf{~ c m}$ & 10 & 4 & 7 & 6 & 7 & 34 \\
\hline $\mathbf{5 0 - 7 9} \mathbf{~ c m}$ & 9 & 18 & 18 & 16 & 10 & 71 \\
\hline $\mathbf{2 8 0} \mathbf{~ c m}$ & 7 & 10 & 10 & 12 & 5 & 44 \\
\hline Total & 26 & 32 & 35 & 35 & 22 & 150 \\
\hline
\end{tabular}

* For one log in decay stage 4 , the diameter at breast height was not recorded.

Most fungi were identified in the field. Interesting and problematic species were collected for further study. Identifications and species concepts of major groups of fungi follow widely accepted monographs, namely Hansen \& Knudsen (2000) for ascomycetes, Knudsen \& Vesterholt (2012) for agarics, Bernicchia \& Gorjón (2010) for corticioid fungi, and Hansen et al. (1997) for other Aphyllophorales. In several cases, specialised monographs of narrower taxonomic groups and specialised scientific papers were used for species not included in the above-listed monographs or for species with recently updated delimitation. Names of fungi and authors' abbreviations follow the databases MycoBank (Wiele \& Stalpers, on-line) and Index Fungorum (Anonymus 2, on-line) in most cases. Voucher specimens are deposited in the public herbaria PRM, SLO and SAV and in the private herbaria of Stanislav Glejdura (PSG) and Vladimír Kunca (PVKU). Abbreviations of all public herbaria are cited in accordance with Index Herbariorum (Thiers, on-line).

Due to the lack of a common European Red list of fungi, some rare and/or threatened species were classified as "species of special interest" (SSI) based on data from several national Red lists (Benkert et al. 1992, Wojewoda \& Ławrynowicz 1992, Arnolds \& Ommering 1996, Stoltze \& Pihl 1998, Rimóczi et al. 1999, Gärdenfors 2010, Holec \& Beran 2006). Indicator species of the nature conservation value of beech forests are adopted from Christensen et al. (2004).

Collection and identification of epiphytic lichens. To study the epiphytic species composition at site level, a number of standing living beech trees was selected by stratified sampling. A few dead beech trees and dead snags were also included. The inventory focused on lichens from the tree base up to a height of $2 \mathrm{~m}$. In order to capture as many species as possible, the phorophytes were taken from substrates differing in the following features: (1) trunk size class according to diameter at breast height $(1.3 \mathrm{~m}$ above the ground, class $1=20-49 \mathrm{~cm}$, class $2=50-79 \mathrm{~cm}$, class $3=\min .80 \mathrm{~cm}$ ), (2) growth form (normal, damaged by rot, slow growing, high stump), (3) light conditions (gap, closed or open forest, 
forest edge), (4) altitude and exposition range (valley bottom, hilltop, slope exposition), (5) bark structure (cracks, rough, smooth), (6) inclination of the trunk $(0=$ no, 1 = yes $)$, and ( 7 ) moss cover (\% of trunk, $0-2 \mathrm{~m})$. Within each site, usually 21-27 trees were surveyed in 3-5 circular plots (15 m diameter), and different plots were situated at a distance of min. $100 \mathrm{~m}$. The vast majority of trees had developed under rather shaded conditions (e.g. in closed forest).

In total, 152 trees were studied in detail: 21 at Havešová, 25 at Stužica, 24 at Vihorlat, 23 at Oblík, 27 at Dobročský prales, 6 at Badínsky prales, and 26 at Klenovský Vepor (Tab. 2). In addition to the stratified beech tree sampling, extra substrates (rocks, different tree species) were investigated in order to obtain an overall impression of the diversity at each site. The genus Chaenothecopsis Vain., a non-lichenised member of the Mycocaliciaceae was included in the inventory, because many taxa grow in the same habitats as lichens and several are obligately or facultatively lichenicolous (Groner 2006).

Tab. 2. Numbers of beech trunks investigated for lichen diversity in defined growth forms and thicknesses. The data originate from all investigated sites. Missing data from 9 trees (in Havešová and Stužica) explain differences in the total numbers of surveyed trees.

\begin{tabular}{|l|c|c|c|c|c|}
\hline Trunk thickness & normal & damaged by rot & slow growing & high stump & Total \\
\hline $\mathbf{2 0 - 4 9} \mathbf{~ c m}$ & 30 & 15 & 11 & 5 & 61 \\
\hline $\mathbf{5 0 - 7 9} \mathbf{~ c m}$ & 24 & 16 & 3 & 7 & 50 \\
\hline $\mathbf{2 8 0} \mathbf{~ c m}$ & 20 & 11 & 0 & 1 & 32 \\
\hline Total & 74 & 42 & 14 & 13 & 143 \\
\hline
\end{tabular}

Most species were identified in the field using a 10× magnification hand lens (with LED light). Chemical $\mathrm{K}$ and/or $\mathrm{C}$ spot tests were applied on lichens in the field. Some problematic specimens were collected and identified afterwards using a microscope and thin-layer chromatography (TLC). We received help with identification from several experts, namely André Aptroot (Soest, The Netherlands), Ulf Arup (University of Lund, Sweden), Zdeněk Palice (Institute of Botany of the Czech Academy of Sciences, Czech Republic), Jiří Malíček (Charles University in Prague, Czech Republic) and Anna Guttová (Institute of Botany, Slovak Academy of Sciences, Slovakia). Nomenclature of lichens follows Wirth et al. (2013) and the current Slovak lichen checklist (Guttová et al. 2013). Voucher specimens are deposited in SAV and a few also in other public herbaria. Redlisted species are based on the national Slovak Red list (Pišút et al. 2001). Indicator species of ecological continuity of natural montane forests in Slovakia follow the list by Pišút (1997) updated by Lackovičová \& Guttová (2005). 
RESULTS AND DISCUSSION

FUNGI

\section{Summary of results}

During six days of field research in seven nature reserves in Slovakia, we identified 215 species of fungi on fallen beech logs (Tab. 3) and 27 additional species on fallen logs of other trees or on fruitbodies of other wood-inhabiting fungi (Tab. 4). Of the species found on beech wood, 178 species (84\%) were recorded during the detailed surveys of selected logs (Tab. 1). The most frequent species, showing the highest average percentage of occurrence on investigated logs and occurring at all sites, were Fomes fomentarius (64\% of logs), Eutypa spinosa (56.1\%), Kretzschmaria deusta (38.6\%), Stereum hirsutum (35.8\%), Ganoderma applanatum (33\%) and Mycena galericulata (21.3\%).

The two sites in the Poloniny Mts., Stužica with 126 species and Havešová with 121, had a distinctly higher number of recorded species on beech logs compared to other sites (Fig. 2). The relative low number of species recorded at Badínsky prales reflects the fact that this site was visited briefly without detailed log inventories. The remaining four sites have very similar numbers of recorded species on beech logs: 74 at Oblík, 72 at Vihorlat, 80 at Klenovský Vepor, and 75 at Dobročský prales. More than 36\% (77 species) were recorded at a single site and $20 \%$ (44 species) at only two sites.

The number of site-specific species (species recorded at a single site only) quite nearly reflects the total numbers of species recorded per site. The highest number of site-specific species was recorded at the sites in Poloniny Mts. (20 at Havešová and 18 at Stužica) with lower numbers recorded at other sites (8 at Vihorlat, 9 at Oblík, 6 at Klenovský Vepor, and 11 at Dobročský prales). Thirtyfive percent of the records of site-specific species originate from the unstructured surveys of additional substrates. Some species can be denoted as site-selective because they show strong deviances in frequency of occurrence on beech logs at specific sites. This deviance can be positive or negative, compared to the average frequency of the same species across all sites (Tab. 5).

Because of relatively stable weather at the time of visit (dry and hot after a few periods of rain), we think that the species diversity at studied sites was generally below average, especially at sun/wind-exposed sites (e.g. Vihorlat). The highest total fungal species richness at Havešová and Stužica probably reflects (1) their large area, (2) sampling differences (at both sites and Klenovský Vepor 30 investigated logs compared to others with 20 logs only), (3) the high number of site-specific fungi reflecting the high naturalness of the stands. 


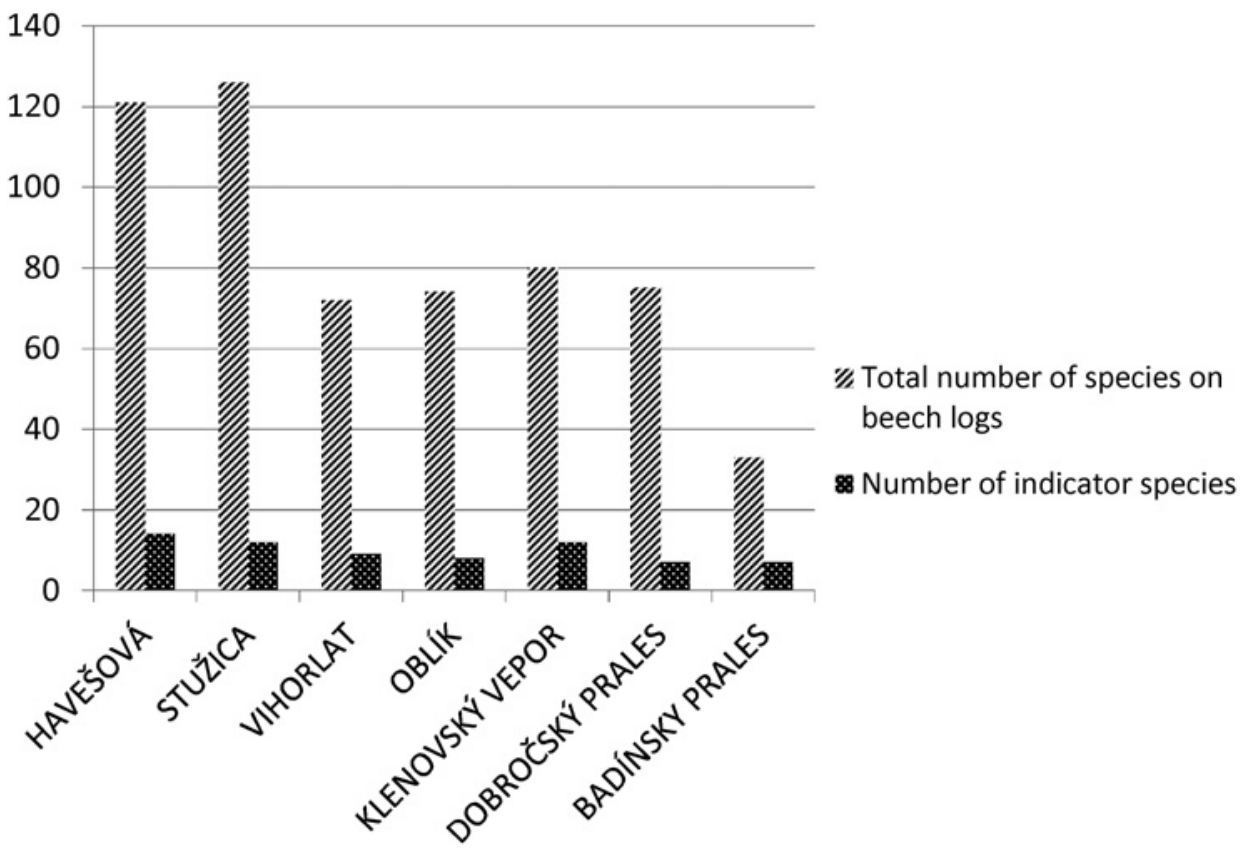

Fig. 2. Comparison of fungal diversity and presence of indicator species on beech logs at investigated sites.

Comparison of average numbers of species per investigated beech log does not show the same pattern as the total fungal species richness at the investigated sites. In this case, Dobročský prales had the highest average diversity per beech log, followed by Stužica and Havešová (Fig. 3). The lower fungal diversity per beech log, low total number of species and low number of site-specific species at Vihorlat, Oblík and Klenovský Vepor probably reflect that these nature reserves are situated at higher altitudes, on tops or ridges of hills. Hence, they are exposed to winds from all directions. Also lacking water in the proximity, e.g. streams and rivers, may have affected fungal fruiting negatively.

\section{New and noteworthy records for Slovakia}

The knowledge on the occurrence of fungi in Slovakia is very unbalanced among various taxonomic groups. The best known group of fungi are polypores thanks to the monograph by Kotlaba (1984). In contrast, records on the occurrence of agarics and ascomycetes have not been compiled in monographic studies and thus the knowledge of their distribution and occurrence varies strongly between regions of Slovakia and is far from complete (Adamčík et al. 2003). 


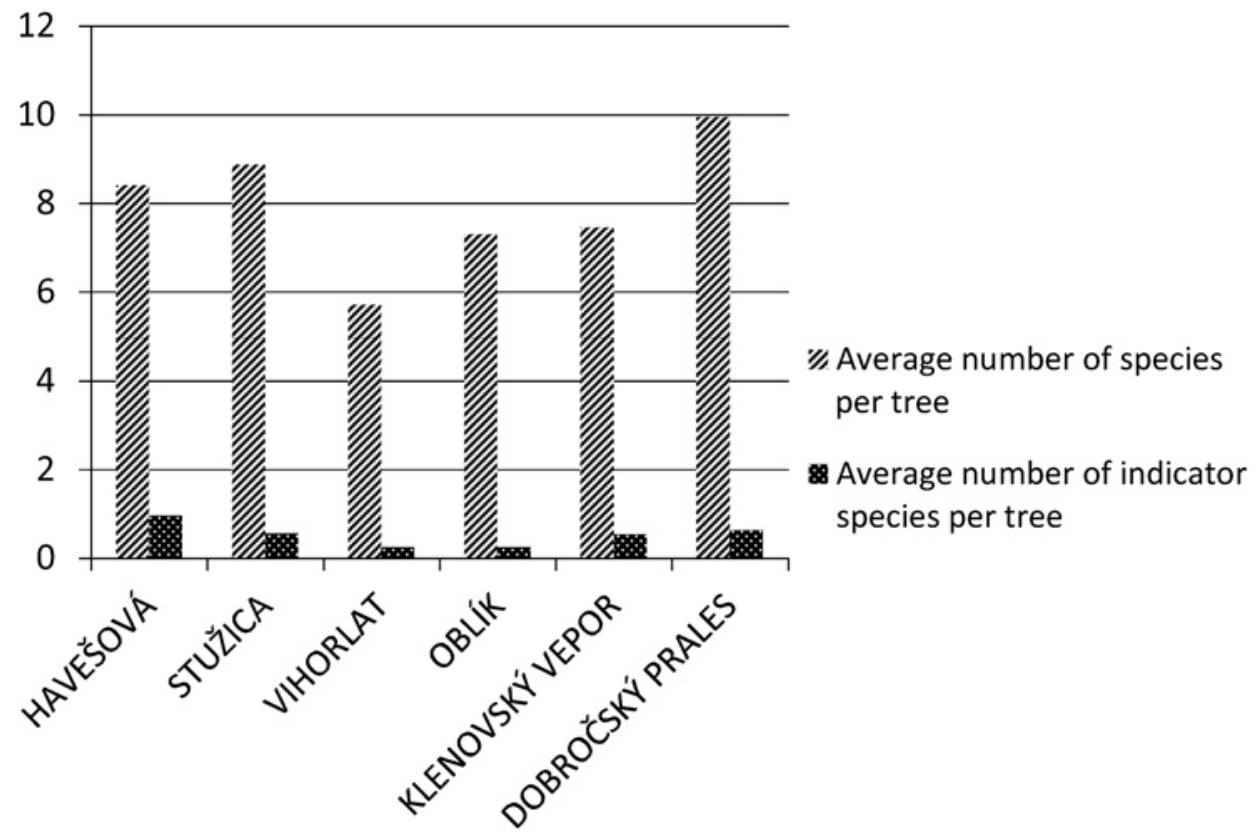

Fig. 3. Comparison of average number of all and indicator species of fungi observed on methodically investigated logs at all investigated sites. Because of limited time, these data were not obtained at Badínsky prales.

We recorded nine species which are probably reported here for the first time in Slovakia. It seems that the least known group of wood-inhabiting fungi in Slovakia are corticioid fungi. Among all 37 recorded corticioid fungi, three species are probably new to Slovakia: Gloeohypochnicium analogum on fallen beech logs, Asterostroma medium on Abies and Hymenochaete ulmicola on Ulmus. Other insufficiently known fungi in Slovakia are ascomycetes with three species new to Slovakia of 41 recorded. Among them two are pyrenomycetes recorded on beech logs: Hypocrea parmastoi and Melanomma spiniferum. The third ascomycete species, Scutellinia colensoi is a very rare discomycete (Schumacher 1990), which within central Europe is only known from the Czech Republic (reported as $S$. badio-berbis by Svrček 1981). Three species of agarics are probably new to Slovakia, namely Entoloma hispidulum, E. pseudoparasiticum, and Hohenbuehelia valesiaca. Crepidotus malachioides, new to Slovakia and meanwhile published by Jančovičová et al. (2014), was also found during the Symposium. Three agarics, Coprinopsis laanii, C. spelaiophila and Galerina autumnalis were reported from Slovakia recently (Hagara 2014) but had not been known from the country at the time of our field research. 
The name Antrodiella pallescens is reported here for the first time in Slovakia, but this species was reported from various parts of the country (e.g. Kotlaba 1984) as A. semisupina (Berk. \& M.A. Curtis) Ryvarden, the latter name corresponding to a taxon occurring exclusively in North America (Miettinen et al. 2006). Galerina autumnalis and Ossicaulis lachnopus are other species not reported from Slovakia until recently because they were treated within the wider taxonomic concepts of G. marginata (Batsch) Kühner and O. lignatilis (Pers.) Redhead \& Ginns, respectively. It is interesting that in Central Europe O. lignatilis tends to occur in man-influenced habitats, whereas O. lachnopus is a distinct but related species associated to more natural habitats (Holec \& Kolařík 2013). This means that O. lignatilis has to be replaced by O. lachnopus in the Slovak Red list (Lizoň 2001).

Two species recorded during our research had not been reported from Slovakia for more than 15 years. One of them, Hemistropharia albocrenulata is included in the Red book and Red list of Slovakia (Antonín et al. 1995, Lizoň 2001). Dobročský prales is the second known locality of Hemistropharia albocrenulata (the first find is from Slovenský raj; Antonín et al. 1995), but this rare species is often recorded in human-influenced habitats (parks etc.) (Holec \& Beran 2006). The other species recollected in Slovakia after more than 15 years is Xylodon pruni (Pilát 1926), possibly overlooked rather than genuinely rare.

Other rare and red-listed species (Lizoň 2001) recorded during our research are Amylocystis lapponica, Flaviporus citrinellus (= Antrodiella citrinella), Oxyporus latemarginatus, Yuchengia narymica, Multiclavula mucida and Tatraea dumbirensis, found on beech logs, and Chrysomphalina grossula observed on wood of a coniferous tree. Amylocystis lapponica is apparently a very rare species in central Europe (Kotlaba 1984) and was included in the candidate list of the European Council for Conservation of Fungi (Dahlberg \& Croneborg 2003). It is now confirmed that it still occurs in the previously only known locality in Slovakia (Dobročský prales), to which we are adding Klenovský Vepor as a new locality for Slovakia. Three other red-listed species were recorded in five to seven of the investigated localities: Camarops tubulina, Ischnoderma resinosum and Stereum subtomentosum. According to our observations, they do not seem to be rare in natural habitats, but at least the first two species appear to be species restricted to such habitats (Holec 2005, Adamčík et al. 2007). Two species from our list are not included in the Slovak Red list (Lizoň 2001) but are included in the Red book by Antonín et al. (1995) as species known from the country: Arrhenia epichysium and Hydropus atramentosus. In our opinion they are rare and typical species of old-growth forests.

Our list of species recorded on beech logs and other dead wood also contains other rare taxa not included in the Slovak Red list (Lizoň 2001). In total, we recorded only only 13 of all 239 species listed in the Red list. This is a low number 
suggesting that rare species of natural beech forests are underestimated in the Slovak Red list. To evaluate the importance of collections of wood-inhabiting fungi during our research, the Czech Red list (Holec \& Beran 2006) seems to be more suitable: it includes 35 species from our list, which is based on a study of the best preserved beech forests in Slovakia and represents a model case for such forests.

Several species reported here are rare, typical of old-growth forests and known from Slovakia only from a few publications (cited in parenthesis for each species) according to the literature and also in our experience: Ceriporiopsis subvermispora (Škubla 1996, Adamčík et al. 2007), Clitocybe truncicola (Adamčík et al. 2007), Clitocybula abundans (Kuthan et al. 1999), C. familia (Antonín et al. 2011), Entoloma jahnii (Jančovičová \& Adamčík 2012), E. placidum (Kuthan et al. 1999), Gymnopilus bellulus (Kuthan et al. 1999), Hohenbuehelia auriscalpium (Adamčík et al. 2007, Glejdura 2013), Ionomidotis irregularis (Kuthan et al. 1999, Adamčík et al. 2007), Lentaria epichnoa (Kuthan et al. 1999), Henningsomyces candidus (Škubla 1998a, Adamčík et al. 2007), Lentinellus ursinus (Ripková et al. 2007, Kotlaba 2000), Pachyella violaceonigra (Kult 1989, Hagara 1989, Škubla 1998b, Kabát et al. 2007), Phlebia nothofagi (Adamčík et al. 2007), Pholiota squarrosoides (Kuthan et al. 1999, Adamčík et al. 2007), Rigidoporus crocatus (Kotlaba 1984), Scutellinia pennsylvanica (Jeannerot 2011), Steccherinum bourdotii (Ripková et al. 2007), S. murashkinskyi (Adamčík et al. 2007) and Tricholomopsis osiliensis (Holec 2012).

Some other species are also known from just a few reports in Slovakia (cited in parenthesis for each species), but their occurrence is insufficiently known and they may be overlooked rather than rare: Camarops polysperma (Kuthan 1984), Cosmospora coccinea (Mihál et al. 2009), Crustomyces subabruptus (Kult 1989, Kuthan et al. 1999), Entoloma tjallingiorum (Kult 1991), Galerina ampullaceocystis (Kuthan \& Singer 1987), Hydnocristella himantia (Fellner 1994), Hypocrea rufa (Tomáň \& Kabát 2004, Mihál \& Blanár 2011), Hypoxylon macrocarpum (Pouzar 1987), Mucronella calva (Svrček 1987), Phanerochaete velutina (Kuthan et al. 1999), Phlebia aurea (Kuthan et al. 1999), P. livida (Kuthan et al. 1999), Pluteus diettrichii (Glejdura 1997), P. hispidulus (Kuthan et al. 1999, Ripková et al. 2007), P. phlebophorus (Kuthan et al. 1999), Porotheleum fimbriatum (Kotlaba et al. 1991, Fellner 1994), Psathyrella olympiana (Kuthan et al. 1999), P. senex (Bolla 1858, Vašutová 2006), Trechispora hymenocystis (Kuthan et al. 1999). Previous collections of Entoloma dichroum (Pers.) P. Kumm. on beech logs from the Poloniny Mts. (incl. one collection from Stužica) identified by S. Adamčík (Kuthan et al. 1999) are possibly identical with a species identified here as E. tjallingiorum.

Many of the species mentioned above as rare or infrequently reported were recently included in a popular book by Hagara (2014). This book illustrates the 
field appearance of fungi mainly based on single Slovak collections. Although it contains valuable data on the diversity and occurrence of fungi in Slovakia, a single cited record for each species does not allow for interpretation of its occurrence in the country.

\section{Indicator species of conservation value of beech forests}

Christensen et al. (2004) introduced an evaluation system of nature conservation value of European beech forests based on presence of indicator species. They listed 21 fungal indicator species. During our field research, we recorded 15 of these. According to our recent data, the highest scores (according to the number of recorded fungal indicators) were established for Havešová (14 indicators), followed by Stužica and Klenovský Vepor (12 indicators each). Nine indicators were scored at Vihorlat, eight at Oblík and seven at the two remaining sites (Fig. 2).

Because the total number of indicator species might be influenced by the number of investigated logs and size of localities, we also compared the average number of indicator species per investigated log for six of the visited sites (Fig. 3). On average at least one indicator was recorded per two logs investigated at Havešová, Stužica and Dobročský prales. In contrast, at least half as many indicator species were recorded at Vihorlat and Oblík.

Among the studied sites, only Havešová and Stužica had previously been investigated for presence of indicator species. Adamčík et al. (2007) reported 13 species from Havešová and 16 from Stužica. Together with the data gathered during our current research, the total score of Havešová is 16 indicators and 19 for Stužica. Published reports of indicator species from the other investigated sites are very scattered in the literature. We were able to locate some additional reports on Camarops tubulina, Gelatoporia pannocincta and Hericium coralloides from Dobročský prales (Glejdura 2002, Antonín et al. 1995) and Ganoderma pfeifferi from Badínsky prales (Kotlaba 1984). This increases the score to 10 indicator species for Dobročský prales and to 8 for Badínsky prales.

Comprehensive data on fungi in beech forest reserves in other parts of $\mathrm{Eu}-$ rope are available but incomplete, nevertheless there is no doubt that the best Slovak sites are of international value for the conservation of wood-inhabiting fungi. This is obvious from the number of indicator species recorded. Already previous to our current investigation, Stužica was the site with the highest number of indicator species (16) according to Christensen et al. (2004). With the addition of three more indicator species this position is confirmed. Detailed investigations of some of the best preserved forest reserves in Ukraine, Romania and in the Balkans, would probably result in similarly high scores. 
Tab. 3. List of all fungal species on beech logs recorded at investigated sites incl. frequencies (as percentage of occurrence on investigated logs).

General abbreviations: N - taxon new to Slovakia, R - rare, interesting or potentially endangered species (species of special interest), I - indicator species of conservation value of beech forests according to Christensen et al. (2004), X - additional records from non-stratified sampling.

Abbreviations of sites: B - Badínsky prales, D - Dobročský prales, H - Havešová, K - Klenovský Vepor, O - Oblík, S - Stužica, V - Vihorlat (for details, see Material and methods).

\begin{tabular}{|c|c|c|c|c|c|c|c|c|c|}
\hline Status & Species & $\mathbf{H}$ & $\mathbf{S}$ & V & $\mathbf{0}$ & $\mathbf{K}$ & D & B & Specimen(s) \\
\hline & $\begin{array}{l}\text { Ampulloclitocybe clavipes (Pers.) Redhead, } \\
\text { Lutzoni, Moncalvo \& Vilgalys }\end{array}$ & & & $\mathrm{X}$ & & & & & \\
\hline & $\begin{array}{l}\text { Annulohypoxylon cohaerens (Pers.) Y.M. Ju, } \\
\text { J.D. Rogers \& H.M. Hsieh }\end{array}$ & $10 \%$ & $17 \%$ & & $35 \%$ & $26 \%$ & $21 \%$ & & SL0 F-1321 (0) \\
\hline & $\begin{array}{l}\text { Annulohypoxylon multiforme (Fr.) Y.M. Ju, } \\
\text { J.D. Rogers \& H.M. Hsieh }\end{array}$ & & & & $5 \%$ & & & & \\
\hline & Antrodia heteromorpha (Fr.) Donk & & & & & & $\mathrm{X}$ & & SAV F-3436 (D) \\
\hline & Antrodiella faginea Vampola \& Pouzar & & & & & $3 \%$ & & & \\
\hline $\mathrm{R}$ & $\begin{array}{l}\text { Antrodiella pallescens (Pilát) } \\
\text { Niemelä \& Miettinen }\end{array}$ & & & $5 \%$ & & & & & \\
\hline \multirow[t]{13}{*}{$\mathrm{R}$} & $\begin{array}{l}\text { Arrhenia epichysium (Pers.) Redhead, } \\
\text { Lutzoni, Moncalvo \& Vilgalys }\end{array}$ & $3 \%$ & $\mathrm{X}$ & & & $\mathrm{X}$ & & & SAV F-4110 (H) \\
\hline & Artomyces pyxidatus (Pers.) Jülich & & $\mathrm{X}$ & & & & & & \\
\hline & Ascocoryne cylichnium (Tul.) Korf & $3 \%$ & $10 \%$ & $5 \%$ & & $10 \%$ & $5 \%$ & & PSG $6386(\mathrm{~S})$ \\
\hline & $\begin{array}{l}\text { Ascocoryne sarcoides (Jacq.) } \\
\text { J.W. Groves \& D.E. Wilson }\end{array}$ & $3 \%$ & $3 \%$ & & & & & & \\
\hline & $\begin{array}{l}\text { Aurantioporus fissilis (Berk. \& M.A. Curtis) } \\
\text { H. Jahn ex Ryvarden }\end{array}$ & $3 \%$ & & & & & & & $\begin{array}{l}\text { SLO F-1320 (H) } \\
\text { PVKU } 233(\mathrm{H})\end{array}$ \\
\hline & Auricularia mesenterica (Dicks.) Pers. & & & & & & $5 \%$ & & \\
\hline & Basidioradulum radula (Pers.) Nobles & $3 \%$ & $10 \%$ & $5 \%$ & $15 \%$ & $10 \%$ & $26 \%$ & & SAV F-3441 (0) \\
\hline & Bisporella citrina (Batsch) Korf \& S.E. Carp. & $\mathrm{X}$ & $\mathrm{X}$ & $10 \%$ & & & $5 \%$ & & \\
\hline & Bjerkandera adusta (Willd.) P. Karst. & $13 \%$ & $3 \%$ & $10 \%$ & $\mathrm{X}$ & $10 \%$ & $32 \%$ & & \\
\hline & Bolbitius reticulatus (Pers.) Rick. & & & & & $3 \%$ & $5 \%$ & & \\
\hline & Bulgaria inquinans (Pers.) Fr. & & & $5 \%$ & $5 \%$ & & & & \\
\hline & Byssomerulius corium (Pers.) Parmasto & & & $5 \%$ & & & & & \\
\hline & Camarops polysperma (Mont.) J.H. Mill. & & $\mathrm{X}$ & & $5 \%$ & & & & SAV F-3440 (0) \\
\hline \multirow[t]{5}{*}{$\mathrm{I}, \mathrm{R}$} & Camarops tubulina (Alb. \& Schwein.) Shear & $3 \%$ & $3 \%$ & $5 \%$ & $\mathrm{X}$ & $3 \%$ & & & $\begin{array}{l}\text { PSG } 4717(0) \\
\text { SAV F-3480 (K) } \\
\text { PRM } 899416(\mathrm{~V}) \\
\text { SL0 F-1280 (S) }\end{array}$ \\
\hline & Catinella olivacea (Batsch) Boud. & & $3 \%$ & $5 \%$ & $10 \%$ & & & & $\begin{array}{l}\text { PVKU } 243(\text { (S) } \\
\text { PSG } 4705 \text { (S) } \\
\text { PSG } 4711(\mathrm{~V}) \\
\text { PSG } 4712(0)\end{array}$ \\
\hline & $\begin{array}{l}\text { Ceraceomyces cf. borealis (Romell) } \\
\text { J. Erikss. \& Ryvarden }\end{array}$ & $\mathrm{X}$ & $\mathrm{X}$ & $5 \%$ & & & & & \\
\hline & Ceriporia excelsa (S. Lundell) Parmasto & & $\mathrm{X}$ & & & & $11 \%$ & $\mathrm{X}$ & $\begin{array}{l}\text { SAV F-4131 (D) } \\
\text { SAV F-4134 (S) }\end{array}$ \\
\hline & Ceriporia reticulata (Hoffm.) Domański & $3 \%$ & & & $\mathrm{X}$ & & & & \\
\hline
\end{tabular}


ADAMČÍK S. ET AL.: FUNGI AND LICHENS OF NATURAL BEECH FORESTS IN SLOVAKIA

\begin{tabular}{|c|c|c|c|c|c|c|c|c|c|}
\hline Status & Species & $\mathbf{H}$ & $\mathbf{S}$ & V & $\mathbf{0}$ & $\mathbf{K}$ & D & B & Specimen(s) \\
\hline & Ceriporia viridans (Berk. \& Broome) Donk & & $\mathrm{X}$ & & $5 \%$ & $3 \%$ & & & \\
\hline I & Ceriporiopsis gilvescens (Bres.) Domański & $27 \%$ & $10 \%$ & $5 \%$ & & $10 \%$ & $11 \%$ & $\mathrm{X}$ & $\begin{array}{l}\text { SAV F-3419 (K) } \\
\text { SAV F-4112 (D) }\end{array}$ \\
\hline \multirow[t]{3}{*}{$\mathrm{R}$} & $\begin{array}{l}\text { Ceriporiopsis subvermispora (Pilát) } \\
\text { Gilb. \& Ryvarden }\end{array}$ & & & & & & $5 \%$ & & SAV F-4121 (D) \\
\hline & Climacocystis borealis (Fr.) Kotl. \& Pouzar & & & & & & $\mathrm{X}$ & & SAV F-3417 (D) \\
\hline & Clitocybe diatreta (Fr.) P. Kumm. & & $3 \%$ & $5 \%$ & & & & & SL0 F-1277 (S) \\
\hline \multirow[t]{18}{*}{$\mathrm{R}$} & Clitocybe truncicola (Peck) Sacc. & & $\mathrm{X}$ & & & & & & PRM 899413 (S) \\
\hline & $\begin{array}{l}\text { Clitopilus hobsonii (Berk. \& Broome) } \\
\text { P.D. Orton }\end{array}$ & $10 \%$ & $7 \%$ & $10 \%$ & & & & & \\
\hline & Coniophora puteana (Schumach.) P. Karst. & $\mathrm{X}$ & & & & & & & \\
\hline & Conocybe subpubescens P.D. Orton & $3 \%$ & $3 \%$ & $5 \%$ & $10 \%$ & & & & \\
\hline & $\begin{array}{l}\text { Coprinellus micaceus (Bull.) } \\
\text { Vilgalys, Hopple \& Jacq. Johnson }\end{array}$ & $13 \%$ & $17 \%$ & & & & $16 \%$ & & \\
\hline & $\begin{array}{l}\text { Coprinopsis laanii (Kits van Wav.) Redhead, } \\
\text { Vilgalys \& Moncalvo }\end{array}$ & & $7 \%$ & & & & & & SAV F-4132 (S) \\
\hline & $\begin{array}{l}\text { Coprinopsis spelaiophila } \\
\text { (Bas \& Uljé) Redhead, Vilgalys \& Moncalvo }\end{array}$ & & & & $\mathrm{X}$ & & & & SAV F-4125 (0) \\
\hline & Crepidotus applanatus (Pers.) P. Kumm. & $\mathrm{X}$ & $13 \%$ & $10 \%$ & $10 \%$ & $13 \%$ & $\mathrm{X}$ & $\mathrm{X}$ & $\begin{array}{l}\text { SL0 F-1259 (H) } \\
\text { SL0 F-1252 (S) } \\
\text { SL0 F-1254 (V) } \\
\text { SL0 F-1318 (0) } \\
\text { SL0 F-1310 (D) } \\
\text { SL0 F-1322 (B) }\end{array}$ \\
\hline & Crepidotus crocophyllus (Berk.) Sacc. & $7 \%$ & $\mathrm{X}$ & & & & & & $\begin{array}{l}\text { SL0 F-1258 (H) } \\
\text { SL0 F-1273 (S) } \\
\text { SAV F-4104 (H) }\end{array}$ \\
\hline & $\begin{array}{l}\text { Crepidotus malachioides Consiglio, Prydiuk } \\
\& \text { Setti }\end{array}$ & $\mathrm{X}$ & $13 \%$ & $5 \%$ & & & & & $\begin{array}{l}\text { SL0 F-1272 (H) } \\
\text { SL0 F-1250 (S) }\end{array}$ \\
\hline & Crepidotus mollis (Schaeff.) Staude & $\mathrm{X}$ & & & & $3 \%$ & & & SL0 F-1266 (H) \\
\hline & $\begin{array}{l}\text { Crustomyces subabruptus } \\
\text { (Bourdot \& Galzin) Jülich }\end{array}$ & & $7 \%$ & $\mathrm{X}$ & & $3 \%$ & $5 \%$ & & \\
\hline & Cyathus striatus (Huds.) Willd. & $3 \%$ & & & & & & & \\
\hline & Cylindrobasidium laeve (Pers.) Chamuris & & $7 \%$ & $5 \%$ & & $3 \%$ & & & \\
\hline & Cystolepiota moelleri Knudsen & $\mathrm{X}$ & & & & & & & SAV F-3437 (H) \\
\hline & Daedaleopsis confragosa (Bolton) J. Schröt. & & $\mathrm{X}$ & & $\mathrm{X}$ & $3 \%$ & & & SL0 F-1315 (0) \\
\hline & $\begin{array}{l}\text { Daedaleopsis confragosa var. tricolor (Bull.) } \\
\text { Bondartsev \& Singer }\end{array}$ & $7 \%$ & & $\mathrm{X}$ & $5 \%$ & & & & SAV F-4106 (0) \\
\hline & Datronia mollis (Sommerf.) Donk & $\mathrm{X}$ & $3 \%$ & $5 \%$ & $10 \%$ & $6 \%$ & $21 \%$ & & SAV F-3455 (D) \\
\hline \multirow[t]{3}{*}{ I } & Dentipellis fragilis (Pers.) Donk & $10 \%$ & $20 \%$ & $10 \%$ & $15 \%$ & $29 \%$ & $11 \%$ & $\mathrm{X}$ & $\begin{array}{l}\text { SAV F-3414 (B) } \\
\text { SAV F-3418 (K) } \\
\text { PRM } 899408 \text { (S) }\end{array}$ \\
\hline & Diatrype disciformis (Hoffm.) Fr. & & & & & $3 \%$ & & & \\
\hline & Entoloma conferendum (Britzelm.) Noordel. & & $\mathrm{X}$ & & & & & & SAV F-4103 (S) \\
\hline $\mathrm{R}$ & Entoloma jahnii Wölfel \& Winterh. & $7 \%$ & & $5 \%$ & & $\mathrm{X}$ & $5 \%$ & & $\begin{array}{l}\text { SL0 F-1291 (V) } \\
\text { SAV F-3439 (K) }\end{array}$ \\
\hline
\end{tabular}


CZech Mycology 68(1): 1-40, February 1, 2016 (ONLINE VERSION, ISSN 1805-1421)

\begin{tabular}{|c|c|c|c|c|c|c|c|c|c|}
\hline Status & Species & $\mathbf{H}$ & $\mathbf{S}$ & $\mathbf{V}$ & $\mathbf{0}$ & $\mathbf{K}$ & D & B & Specimen(s) \\
\hline $\mathrm{R}$ & Entoloma placidum (Fr.) Noordel. & $\mathrm{X}$ & & & & & & & SAV F-4120 (H) \\
\hline \multirow[t]{3}{*}{$\mathrm{N}$} & Entoloma pseudoparasiticum Noordel. & & $3 \%$ & & & & & & SL0 F-1298 (S) \\
\hline & Entoloma tjallingiorum Noordel. & $3 \%$ & & & & $3 \%$ & & & SAV F-4128 (H) \\
\hline & Eutypa spinosa (Pers.) Tul. \& C. Tul. & $50 \%$ & $40 \%$ & $40 \%$ & $65 \%$ & $68 \%$ & $74 \%$ & $\mathrm{X}$ & SL0 F-1279 (S) \\
\hline $\mathrm{I}$ & Flammulaster limulatus (Fr.) Watling & & $3 \%$ & $5 \%$ & & $\mathrm{X}$ & & & $\begin{array}{c}\text { SAV F-4108 (V) } \\
\text { SL0 F-1268 (V) } \\
\text { PVKU } 239(\mathrm{~S})\end{array}$ \\
\hline $\mathrm{I}$ & Flammulaster muricatus (Fr.) Watling & $7 \%$ & $3 \%$ & $\mathrm{X}$ & & $\mathrm{X}$ & & & $\begin{array}{l}\text { SL0 F-1274 (S) } \\
\text { SL0 F-1275 (V) } \\
\text { SAV F-3477 (K) }\end{array}$ \\
\hline \multirow[t]{9}{*}{$\mathrm{R}$} & \begin{tabular}{|l} 
Flaviporus citrinellus \\
(Niemelä \& Ryvarden) Ginns
\end{tabular} & & & & & $3 \%$ & & & SAV F-4122 (K) \\
\hline & Fomes fomentarius (L.) Fr. & $40 \%$ & $60 \%$ & $65 \%$ & $70 \%$ & $65 \%$ & $84 \%$ & $\mathrm{X}$ & \\
\hline & Fomitopsis pinicola (Sw.) P. Karst. & $10 \%$ & $10 \%$ & $5 \%$ & $10 \%$ & $16 \%$ & $5 \%$ & & \\
\hline & Fuscoporia ferruginosa (Schrad.) Murrill & & & $\mathrm{X}$ & & & & $\mathrm{X}$ & SAV F-3433 (B) \\
\hline & Galerina ampullaceocystis P.D. Orton & & & $5 \%$ & & & & & SAV F-4126 (V) \\
\hline & $\begin{array}{l}\text { Galerina autumnalis (Peck) } \\
\text { A.H. Sm. \& Singer }\end{array}$ & & & $\mathrm{X}$ & & & & & PRM 899417 (V) \\
\hline & Galerina marginata (Batsch) Kühner & $3 \%$ & & $\mathrm{X}$ & $10 \%$ & & & & $\begin{array}{l}\text { PRM } 899417(\mathrm{~V}, \\
\text { as } \\
\text { G. autumnalis) }\end{array}$ \\
\hline & Galerina triscopa (Fr.) Kühner & & $3 \%$ & & & & & & SAV F-3468 (S) \\
\hline & Ganoderma applanatum (Pers.) Pat. & $27 \%$ & $40 \%$ & $35 \%$ & $20 \%$ & $29 \%$ & $47 \%$ & $\mathrm{X}$ & \\
\hline I & Gelatoporia pannocincta (Romell) Niemelä & $\mathrm{X}$ & $3 \%$ & $\mathrm{X}$ & $\mathrm{X}$ & $3 \%$ & & $\mathrm{X}$ & $\begin{array}{l}\text { SAV F-3423 (S) } \\
\text { SAV F-3422 (0) } \\
\text { SAV F-3420 (K) }\end{array}$ \\
\hline \multirow[t]{4}{*}{$\mathrm{N}$} & $\begin{array}{l}\text { Gloeohypochnicium analogum } \\
\text { (Bourdot \& Galzin) Hjortstam }\end{array}$ & $3 \%$ & & & & & & & \\
\hline & Gymnopilus penetrans (Fr.) Murrill & & $3 \%$ & & & & & & \\
\hline & Helvella macropus (Pers.) P. Karst. & & $7 \%$ & & & & & & \\
\hline & Helvella lacunosa Afzel. & & & & & & $5 \%$ & & \\
\hline \multirow[t]{2}{*}{$\mathrm{R}$} & Henningsomyces candidus (Pers.) Kuntze & $3 \%$ & & & & & & & \\
\hline & Hericium cirrhatum (Pers.) Nikol. & & & & $5 \%$ & & & & SL0 F-1309 (0) \\
\hline I & Hericium coralloides (Scop.) Pers. & $3 \%$ & & $\mathrm{X}$ & $\mathrm{X}$ & $\mathrm{X}$ & & $\mathrm{X}$ & \\
\hline $\mathrm{I}, \mathrm{R}$ & $\begin{array}{l}\text { Hohenbuehelia auriscalpium (Maire) } \\
\text { Singer }\end{array}$ & $7 \%$ & & & & & $5 \%$ & & SL0 F-1306 (D) \\
\hline \multirow[t]{5}{*}{$\mathrm{N}$} & Hohenbuehelia valesiaca (Ces.) Singer & & $\mathrm{X}$ & & & & & & SAV F-4113 (S) \\
\hline & Humaria hemisphaerica (Hoffm.) Fuckel & $3 \%$ & $3 \%$ & & & $3 \%$ & & & $\begin{array}{l}\text { PSG } 4691(\mathrm{H}) \\
\text { PSG } 4700(\mathrm{~S})\end{array}$ \\
\hline & $\begin{array}{l}\text { Hydnocristella himantia (Schwein.) } \\
\text { R.H. Petersen }\end{array}$ & & & $5 \%$ & & & & & SL0 F-1263 (V) \\
\hline & Hypholoma fasciculare (Huds.) P. Kumm. & $3 \%$ & $10 \%$ & $5 \%$ & & $3 \%$ & $5 \%$ & & \\
\hline & Hypholoma lateritium (Schaeff.) P. Kumm. & & $3 \%$ & & & & & & \\
\hline \multirow[t]{2}{*}{$\mathrm{N}$} & Hypocrea parmastoi Overton & & & & $\mathrm{X}$ & & & & SAV F-4124 (0) \\
\hline & Hypocrea rufa (Pers.) Fr., sensu lato & & $3 \%$ & & & & & & \\
\hline
\end{tabular}


ADAMČÍK S. ET AL.: FUNGI AND LICHENS OF NATURAL BEECH FORESTS IN SLOVAKIA

\begin{tabular}{|c|c|c|c|c|c|c|c|c|c|}
\hline Status & Species & $\mathbf{H}$ & $\mathbf{S}$ & $\mathbf{V}$ & $\mathbf{0}$ & $\mathbf{K}$ & D & B & Specimen(s) \\
\hline & Hypoxylon fragiforme (Pers.) J. Kickx f. & $17 \%$ & $10 \%$ & $10 \%$ & $25 \%$ & $6 \%$ & $32 \%$ & & \\
\hline & Hypoxylon macrocarpum Pouzar & & $3 \%$ & & $5 \%$ & $\mathrm{X}$ & & & \\
\hline & Hypoxylon rubiginosum (Pers.) Fr. & & $3 \%$ & & & & $11 \%$ & & \\
\hline & Inocybe petiginosa (Fr.) Gillet & $3 \%$ & $7 \%$ & & & & & & \\
\hline \multirow[t]{3}{*}{ I } & Inonotus cuticularis (Bull.) P. Karst. & $\mathrm{X}$ & & & & & $\mathrm{X}$ & & SAV F-3454 (D) \\
\hline & Inonotus nodulosus (Fr.) P. Karst. & & & $\mathrm{X}$ & & & & & SAV F-3449 (V) \\
\hline & Inonotus obliquus (Ach. ex Pers.) Pilát & & $7 \%$ & & $5 \%$ & & & & \\
\hline $\mathrm{R}$ & $\begin{array}{l}\text { Ionomidotis irregularis (Schwein.) } \\
\text { E.J. Durand }\end{array}$ & & & $5 \%$ & & & & & PVKU 248 (V) \\
\hline \multirow[t]{6}{*}{$\mathrm{I}, \mathrm{R}$} & Ischnoderma resinosum (Schrad.) P. Karst. & $23 \%$ & $7 \%$ & $\mathrm{X}$ & $5 \%$ & $\mathrm{X}$ & $16 \%$ & $\mathrm{X}$ & \\
\hline & $\begin{array}{l}\text { Junghuhnia lacera (P. Karst.) } \\
\text { Niemelä \& Kinnunen }\end{array}$ & $3 \%$ & & & & & & & \\
\hline & Junghuhnia nitida (Pers.) Ryvarden & & & $\mathrm{X}$ & & & & & \\
\hline & Kretzschmaria deusta (Hoffm.) P.M.D. Martin & $47 \%$ & $50 \%$ & $25 \%$ & $40 \%$ & $23 \%$ & $47 \%$ & $\mathrm{X}$ & \\
\hline & $\begin{array}{l}\text { Laetiporus sulphureus (Bull.) } \\
\text { Bondartsev \& Singer }\end{array}$ & & $\mathrm{X}$ & $\mathrm{X}$ & & & & & \\
\hline & Laxitextum bicolor (Pers.) Lentz & $\mathrm{X}$ & & & & & & & \\
\hline $\mathrm{R}$ & Lentaria epichnoa (Fr.) Corner & & $3 \%$ & & & & & & SAV F-4109 (S) \\
\hline \multirow[t]{9}{*}{$\mathrm{I}, \mathrm{R}$} & Lentinellus ursinus (Fr.) Kühner & $3 \%$ & $\mathrm{X}$ & & & $\mathrm{X}$ & & & SAV F-4115 (S) \\
\hline & Lentinus strigosus Fr. & $3 \%$ & & & & & & & \\
\hline & Lenzites betulina (L.) Fr. & $3 \%$ & & & & $6 \%$ & & & \\
\hline & Lycoperdon nigrescens Wahlenb. & & & & & & $5 \%$ & & \\
\hline & Lycoperdon perlatum Pers. & & $\mathrm{X}$ & & & $3 \%$ & & $\mathrm{X}$ & \\
\hline & Lycoperdon pyriforme Schaeff. & $7 \%$ & $13 \%$ & $10 \%$ & & $10 \%$ & $21 \%$ & & \\
\hline & Marasmius rotula (Scop.) Fr. & $3 \%$ & $3 \%$ & & & & & & \\
\hline & Marasmius torquescens Quél. & & $3 \%$ & & & & & & \\
\hline & $\begin{array}{l}\text { Megacollybia platyphylla (Pers.) } \\
\text { Kotl. \& Pouzar }\end{array}$ & & & & $5 \%$ & & $5 \%$ & & \\
\hline \multirow[t]{14}{*}{$\mathrm{N}$} & Melanomma spiniferum Ellis \& Everh. & & & & & $3 \%$ & & $\mathrm{X}$ & \\
\hline & $\begin{array}{l}\text { Mensularia nodulosa (Fr.) } \\
\text { T. Wagner \& M. Fisch. }\end{array}$ & & $\mathrm{X}$ & & $10 \%$ & $10 \%$ & $5 \%$ & & \\
\hline & Mucronella calva (Alb. \& Schwein.) Fr. & & & & & & $5 \%$ & & SAV F-3471 (D) \\
\hline & Mycena amicta (Fr.) Quél. & $3 \%$ & & & & & & & \\
\hline & Mycena arcangeliana Bres. & $7 \%$ & $13 \%$ & $15 \%$ & $15 \%$ & $23 \%$ & $5 \%$ & & SL0 F-1253 (V) \\
\hline & Mycena cinerella (P. Karst.) P. Karst. & & $3 \%$ & & & & & $\mathrm{X}$ & \\
\hline & Mycena crocata (Schrad.) P. Kumm. & $23 \%$ & $7 \%$ & $5 \%$ & $15 \%$ & & $16 \%$ & $\mathrm{X}$ & \\
\hline & Mycena galericulata (Scop.) Gray & $7 \%$ & $20 \%$ & $50 \%$ & $5 \%$ & $35 \%$ & $11 \%$ & & \\
\hline & Mycena haematopus (Pers.) P. Kumm. & $17 \%$ & $23 \%$ & $15 \%$ & $5 \%$ & $10 \%$ & $5 \%$ & & \\
\hline & Mycena pura (Pers.) P. Kumm. & $3 \%$ & $3 \%$ & & & & & & \\
\hline & Mycena renati Quél. & & $\mathrm{X}$ & & $5 \%$ & & & & \\
\hline & Mycetinis alliaceus (Jacq.) Earle & $13 \%$ & $17 \%$ & $5 \%$ & $25 \%$ & $16 \%$ & $16 \%$ & & \\
\hline & Nemania atropurpurea (Fr.) Pouzar & & & & & $3 \%$ & & & SAV F-4105 (K) \\
\hline & Nemania carbonacea Pouzar & $\mathrm{X}$ & & & & & & & \\
\hline
\end{tabular}


CZech Mycology 68(1): 1-40, February 1, 2016 (ONLINE VERSION, ISSN 1805-1421)

\begin{tabular}{|c|c|c|c|c|c|c|c|c|c|}
\hline Status & Species & $\mathbf{H}$ & $\mathbf{S}$ & $\mathbf{V}$ & $\mathbf{0}$ & $\mathbf{K}$ & D & B & Specimen(s) \\
\hline & Nemania serpens (Pers.) Gray & $3 \%$ & $7 \%$ & $5 \%$ & $5 \%$ & $3 \%$ & $5 \%$ & & \\
\hline & $\begin{array}{l}\text { Neonectria coccinea (Pers.) } \\
\text { Rossman \& Samuels }\end{array}$ & & $7 \%$ & $5 \%$ & $5 \%$ & & & & \\
\hline \multirow[t]{2}{*}{$\mathrm{I}, \mathrm{R}$} & Ossicaulis lachnopus (Fr.) Contu & $\mathrm{X}$ & $3 \%$ & $\mathrm{X}$ & $\mathrm{X}$ & $6 \%$ & $5 \%$ & $\mathrm{X}$ & $\begin{array}{l}\text { PRM } 899407 \text { (S) } \\
\text { PRM } 899418(V)\end{array}$ \\
\hline & Oudemansiella mucida (Schrad.) Höhn. & $17 \%$ & $13 \%$ & $5 \%$ & & $29 \%$ & $11 \%$ & & \\
\hline \multirow[t]{3}{*}{$\mathrm{R}$} & $\begin{array}{l}\text { Oxyporus latemarginatus (Durieu \& Mont.) } \\
\text { Donk }\end{array}$ & $3 \%$ & & & & & & & SAV F-4118 (H) \\
\hline & Oxyporus populinus (Schumach.) Donk & $3 \%$ & & $\mathrm{X}$ & $\mathrm{X}$ & & & & PRM 899414 (V) \\
\hline & Pachyella babingtonii (Berk. \& Broome) Boud. & $\mathrm{X}$ & & & & & & & PSG $4688(\mathrm{H})$ \\
\hline \multirow[t]{10}{*}{$\mathrm{R}$} & Pachyella violaceonigra (Rehm) Pfister & $\mathrm{X}$ & & & & & & & PVKU $235(\mathrm{H})$ \\
\hline & Panellus stipticus (Bull.) P. Karst. & $7 \%$ & $20 \%$ & & $5 \%$ & $6 \%$ & $11 \%$ & & \\
\hline & $\begin{array}{l}\text { Peniophorella praetermissa (P. Karst.) } \\
\text { K.H. Larss. }\end{array}$ & & & & & & $5 \%$ & & \\
\hline & Peziza micropus Pers. & $10 \%$ & $7 \%$ & $\mathrm{X}$ & $10 \%$ & & $11 \%$ & & \\
\hline & Peziza saniosa Schrad. ex J.F. Gmel. & $3 \%$ & & & & & & & PSG $4692(\mathrm{H})$ \\
\hline & Peziza varia (Hedw.) Alb. \& Schwein. & $\mathrm{X}$ & $\mathrm{X}$ & & & & & & \\
\hline & Phanerochaete velutina (DC.) Parmasto & & & & & & $5 \%$ & & \\
\hline & Phlebia aurea (Fr.) Nakasone & & $\mathrm{X}$ & & & & & & \\
\hline & Phlebia centrifuga P. Karst. & & $3 \%$ & & & $\mathrm{X}$ & & & \\
\hline & Phlebia livida (Pers.) Bres. & & $10 \%$ & & & $3 \%$ & & & SAV F-3438 (K) \\
\hline \multirow[t]{6}{*}{$\mathrm{I}, \mathrm{R}$} & Phlebia nothofagi (G. Cunn.) Nakasone & $7 \%$ & $\mathrm{X}$ & & $5 \%$ & $3 \%$ & & & $\begin{array}{l}\text { SAV F-3444 (S) } \\
\text { SAV F-3410 (K) }\end{array}$ \\
\hline & Phlebia rufa (Pers.) M.P. Christ. & & $7 \%$ & $10 \%$ & & & $5 \%$ & & SAV F-3434 (D) \\
\hline & $\begin{array}{l}\text { Phlebia tremellosa (Schrad.) } \\
\text { Nakasone \& Burds. }\end{array}$ & $3 \%$ & $7 \%$ & $10 \%$ & $10 \%$ & $6 \%$ & & & \\
\hline & Phleogena faginea (Fr. \& Palmquist) Link & & $\mathrm{X}$ & & & $3 \%$ & & & \\
\hline & Pholiota adiposa (Batsch) P. Kumm. & & & & & & $5 \%$ & $\mathrm{X}$ & \\
\hline & Pholiota squarrosa (Weigel) P. Kumm. & & & & & & $\mathrm{X}$ & & SAV F-3457 (D) \\
\hline \multirow[t]{10}{*}{$\mathrm{I}, \mathrm{R}$} & Pholiota squarrosoides (Peck) Sacc. & $3 \%$ & $\mathrm{X}$ & & $\mathrm{X}$ & $\mathrm{X}$ & $16 \%$ & & $\begin{array}{l}\text { SL0 F-1256 (S) } \\
\text { SAV F-3412 (K) } \\
\text { SAV F-4107 (0) }\end{array}$ \\
\hline & Pholiotina brunnea (Watling) Singer & $3 \%$ & $3 \%$ & & & $\mathrm{X}$ & & & SAV F-3479 (K) \\
\hline & Phyllotopsis nidulans (Pers.) Singer & & & & $5 \%$ & & & & SL0 F-1309 (0) \\
\hline & Pleurotus dryinus (Pers.) P. Kumm. & $\mathrm{X}$ & & & & & & $\mathrm{X}$ & \\
\hline & Pleurotus pulmonarius (Fr.) Quél. & $17 \%$ & $3 \%$ & & $15 \%$ & $19 \%$ & $37 \%$ & & SAV F-3482 (0) \\
\hline & Plicaturopsis crispa (Pers.) D.A. Reid & & $3 \%$ & & & $6 \%$ & $5 \%$ & $\mathrm{X}$ & \\
\hline & Pluteus cervinus (Schaeff.) P. Kumm. & $17 \%$ & $3 \%$ & $10 \%$ & $10 \%$ & $10 \%$ & $26 \%$ & $\mathrm{X}$ & $\begin{array}{l}\text { SL0 F-1257 (V) } \\
\text { SL0 F-1317 (B) }\end{array}$ \\
\hline & Pluteus chrysophaeus (Schaeff.) Quél. & $3 \%$ & $7 \%$ & & & & $5 \%$ & & $\begin{array}{l}\text { SLO F-1296 (H) } \\
\text { SL0 F-1282 (S) }\end{array}$ \\
\hline & Pluteus diettrichii Bres. & & $\mathrm{X}$ & & & & & & \\
\hline & Pluteus hispidulus (Fr.) Gillet & $\mathrm{X}$ & $7 \%$ & & & & $5 \%$ & & $\begin{array}{l}\text { SL0 F-1300 (S) } \\
\text { PSG } 4696(\mathrm{H})\end{array}$ \\
\hline
\end{tabular}


ADAMČÍK S. ET AL.: FUNGI AND LICHENS OF NATURAL BEECH FORESTS IN SLOVAKIA

\begin{tabular}{|c|c|c|c|c|c|c|c|c|c|}
\hline Status & Species & $\mathrm{H}$ & $\mathbf{S}$ & V & $\mathbf{0}$ & $\mathbf{K}$ & D & B & Specimen(s) \\
\hline & Pluteus leoninus (Schaeff.) P. Kumm. & $7 \%$ & & & & & & $\mathrm{X}$ & \\
\hline & Pluteus luctuosus Boud. & & $3 \%$ & & & & $5 \%$ & & SL0 F-1294 (S) \\
\hline & Pluteus nanus (Pers.) P. Kumm. & $3 \%$ & $3 \%$ & $5 \%$ & $5 \%$ & & & & PRM 899415 (V) \\
\hline & Pluteus petasatus (Fr.) Gillet & & & & $\mathrm{X}$ & & & & PRM $899419(0)$ \\
\hline & Pluteus phlebophorus (Ditmar) P. Kumm. & $\mathrm{X}$ & $3 \%$ & & & & & & \\
\hline & Pluteus plautus (Weinm.) Gillet & $7 \%$ & $3 \%$ & & & $3 \%$ & & & SL0 F-1293 (S) \\
\hline & Pluteus podospileus Sacc. \& Cub. & $7 \%$ & $3 \%$ & & & & $5 \%$ & $\mathrm{X}$ & $\begin{array}{l}\text { SL0 F-1303 (S) } \\
\text { SL0 F-1313 (D) }\end{array}$ \\
\hline & Pluteus salicinus (Pers.) P. Kumm. & $3 \%$ & & & $\mathrm{X}$ & & & & PVKU $232(\mathrm{H})$ \\
\hline & Pluteus semibulbosus (Lasch) Quél. & $7 \%$ & $\mathrm{X}$ & & & & & & SL0 F-1289 (S) \\
\hline & Pluteus thomsonii (Berk. \& Broome) Dennis & & & & & & $\mathrm{X}$ & & SL0 F-1319 (D) \\
\hline I & Pluteus umbrosus (Pers.) P. Kumm. & $3 \%$ & $3 \%$ & & & & & $\mathrm{X}$ & $\begin{array}{l}\text { PSG } 4689(\mathrm{H}) \\
\text { PSG } 4703(\mathrm{~S})\end{array}$ \\
\hline & Polyporus badius (Pers.) Schwein. & $3 \%$ & $\mathrm{X}$ & & & & $11 \%$ & & \\
\hline & Polyporus melanopus (Pers.) Fr. & & & & $5 \%$ & & & & SAV F-3452 (0) \\
\hline & Polyporus squamosus (Huds.) Fr. & $3 \%$ & $\mathrm{X}$ & & $\mathrm{X}$ & & & $\mathrm{X}$ & \\
\hline & Polyporus varius (Pers.) Fr. & $3 \%$ & $10 \%$ & & & $23 \%$ & $11 \%$ & $\mathrm{X}$ & \\
\hline & Porotheleum fimbriatum (Pers.) Fr. & $3 \%$ & & & & & $\mathrm{X}$ & & SAV F-3435 (D) \\
\hline & Postia alni Niemelä \& Vampola & & $3 \%$ & & & & & & \\
\hline & Postia undosa (Peck) Jülich & & & & & $\mathrm{X}$ & & $\mathrm{X}$ & SAV F-4136 (K) \\
\hline & Protomerulius caryae (Schwein.) Ryvarden & $7 \%$ & $7 \%$ & $10 \%$ & $5 \%$ & $3 \%$ & & & $\begin{array}{l}\text { SAV F-3445 (S) } \\
\text { SAV F-3421 (0) } \\
\text { SAV F-3461 (K) }\end{array}$ \\
\hline & Psathyrella fagetophila Örstadius \& Enderle & & & & $5 \%$ & & & & \\
\hline & Psathyrella obtusata (Pers.) A.H. Sm. & $3 \%$ & $3 \%$ & & & & & & \\
\hline & Psathyrella olympiana A.H. Sm. & $\mathrm{X}$ & & & & & & $\mathrm{X}$ & SAV F-4135 (H) \\
\hline & Psathyrella piluliformis (Bull.) P.D. Orton & $3 \%$ & $17 \%$ & $15 \%$ & & $6 \%$ & $11 \%$ & & \\
\hline & Psathyrella senex (Peck) A.H. Sm. & & & & $\mathrm{X}$ & & & & SAV F-3478 (0) \\
\hline & Psathyrella silvestris (Gillet) Konrad \& Maubl. & $3 \%$ & & & & & & & SAV F-4116 (H) \\
\hline & Pycnoporus cinnabarinus (Jacq.) P. Karst. & $7 \%$ & & & & & & & \\
\hline & Ramaria stricta (Pers.) Quél. & $3 \%$ & & & & & & & \\
\hline $\mathrm{R}$ & Rigidoporus crocatus (Pat.) Ryvarden & & $3 \%$ & & & & & $\mathrm{X}$ & $\begin{array}{l}\text { SAV F-3415 (S) } \\
\text { SAV F-3448 (B) } \\
\text { PRM } 899412 \text { (S) }\end{array}$ \\
\hline & $\begin{array}{l}\text { Rigidoporus sanguinolentus } \\
\text { (Alb. \& Schwein.) Donk }\end{array}$ & $10 \%$ & $20 \%$ & & $5 \%$ & $3 \%$ & & & $\begin{array}{l}\text { SAV F-3446 (S) } \\
\text { SAV F-3411 (K) }\end{array}$ \\
\hline & Sarcomyxa serotina (Schrad.) P. Karst. & & $3 \%$ & & & $10 \%$ & $5 \%$ & & \\
\hline & Schizophyllum commune Fr. & $13 \%$ & & & & $6 \%$ & & & \\
\hline & $\begin{array}{l}\text { Schizopora flavipora } \\
\text { (Berk. \& M.A. Curtis ex Cooke) Ryvarden }\end{array}$ & & & & $5 \%$ & & & & \\
\hline & Schizopora paradoxa (Schrad.) Donk & & & & & $3 \%$ & & & \\
\hline & Scutellinia cejpii (Velen.) Svrček & $3 \%$ & $\mathrm{X}$ & & $5 \%$ & & & & $\begin{array}{l}\text { PSG } 4301(\mathrm{H}) \\
\text { PSG } 4308(\mathrm{~S}) \\
\end{array}$ \\
\hline $\mathrm{N}$ & Scutellinia colensoi Massee ex Le Gal & & & $\mathrm{X}$ & & & & & PSG 4281 (V) \\
\hline
\end{tabular}


CZech Mycology 68(1): 1-40, February 1, 2016 (ONLINE VERSion, ISSN 1805-1421)

\begin{tabular}{|c|c|c|c|c|c|c|c|c|c|}
\hline Status & Species & $\mathbf{H}$ & $\mathbf{S}$ & V & $\mathbf{0}$ & $\mathbf{K}$ & D & B & Specimen(s) \\
\hline \multirow[t]{4}{*}{$\mathrm{R}$} & Scutellinia pennsylvanica (Seaver) Denison & $\mathrm{X}$ & $3 \%$ & & & & & & $\begin{array}{l}\text { PSG } 4297(\mathrm{H}) \\
\text { PSG } 4282(\mathrm{~S})\end{array}$ \\
\hline & $\begin{array}{l}\text { Scytinostroma portentosum } \\
\text { (Berk. \& M.A. Curtis) Donk }\end{array}$ & & & & & $\mathrm{X}$ & & & \\
\hline & Simocybe centunculus (Fr.) Singer & $13 \%$ & $17 \%$ & $5 \%$ & $5 \%$ & & $11 \%$ & $\mathrm{X}$ & SL0 F-1290 (H) \\
\hline & Skeletocutis nivea (Jungh.) Jean Keller & $3 \%$ & $3 \%$ & & $5 \%$ & $3 \%$ & & & $\begin{array}{l}\text { SAV F-3416 (S) } \\
\text { SAV F-4111 (0) }\end{array}$ \\
\hline $\mathrm{R}$ & Steccherinum bourdotii Saliba \& A. David & & & & $5 \%$ & & & & \\
\hline \multirow[t]{4}{*}{$\mathrm{R}$} & $\begin{array}{l}\text { Steccherinum murashkinskyi (Burt) } \\
\text { Maas Geest. }\end{array}$ & $3 \%$ & & & & & & & PVKU $237(\mathrm{H})$ \\
\hline & Steccherinum ochraceum (Pers.) Gray & $17 \%$ & $7 \%$ & $5 \%$ & & $6 \%$ & $21 \%$ & & \\
\hline & Stereum hirsutum (Willd.) Pers. & $27 \%$ & $33 \%$ & $25 \%$ & $45 \%$ & $32 \%$ & $53 \%$ & & \\
\hline & Stereum rugosum Pers. & & $3 \%$ & & $5 \%$ & & & & \\
\hline \multirow[t]{2}{*}{$\mathrm{R}$} & Stereum subtomentosum Pouzar & $\mathrm{X}$ & $3 \%$ & $5 \%$ & $5 \%$ & & $11 \%$ & & \\
\hline & Stropharia cyanea (Bull.) Tuomikoski & & $3 \%$ & & & & & & \\
\hline \multirow[t]{14}{*}{$\mathrm{R}$} & Tatraea dumbirensis (Velen.) Svrček & & $\mathrm{X}$ & & & & & & PSG 4701 (S) \\
\hline & Trametes gibbosa (Pers.) Fr. & $17 \%$ & $10 \%$ & $\mathrm{X}$ & $5 \%$ & $16 \%$ & $21 \%$ & $\mathrm{X}$ & \\
\hline & Trametes hirsuta (Wulfen) Lloyd & $17 \%$ & & & $5 \%$ & $10 \%$ & & & \\
\hline & Trametes versicolor (L.) Lloyd & $23 \%$ & $20 \%$ & $5 \%$ & $15 \%$ & $10 \%$ & $32 \%$ & & \\
\hline & Trametopsis cervina (Schwein.) Tomšovský & $\mathrm{X}$ & $3 \%$ & & $10 \%$ & & & & \\
\hline & $\begin{array}{l}\text { Trechispora hymenocystis } \\
\text { (Berk. \& Broome) K.H. Larss. }\end{array}$ & $3 \%$ & & $10 \%$ & & $19 \%$ & $\mathrm{X}$ & & SAV F-3483 (D) \\
\hline & Trechispora mollusca (Pers.) Liberta & & & & & $\mathrm{X}$ & & & SAV F-4133 (K) \\
\hline & Trichaptum abietinum (Pers. ex J.F. Gmel.) Fr. & & & & & & $5 \%$ & & \\
\hline & Trichaptum biforme (Fr.) Ryvarden & $23 \%$ & $7 \%$ & & $30 \%$ & & & & \\
\hline & Tyromyces chioneus (Fr.) P. Karst. & & & $5 \%$ & & $\mathrm{X}$ & & & \\
\hline & Volvariella bombycina (Schaeff.) Singer & $\mathrm{X}$ & & & & & & & \\
\hline & Xerula radicata (Relhan) Dörfelt & $\mathrm{X}$ & $3 \%$ & & $\mathrm{X}$ & $3 \%$ & & & \\
\hline & Xylaria hypoxylon (L.) Grev. & $\mathrm{X}$ & $13 \%$ & & & & $16 \%$ & & \\
\hline & Xylaria polymorpha (Pers.) Grev. & $3 \%$ & $3 \%$ & $5 \%$ & & & $5 \%$ & $\mathrm{X}$ & \\
\hline $\mathrm{R}$ & $\begin{array}{l}\text { Xylodon pruni (Lasch) } \\
\text { Hjortstam \& Ryvarden }\end{array}$ & & & & & & $5 \%$ & $\mathrm{X}$ & SAV F-4123 (D) \\
\hline $\mathrm{R}$ & $\begin{array}{l}\text { Yuchengia narymica (Pilát) } \\
\text { B.K. Cui, C.L. Zhao \& Steffen }\end{array}$ & $\mathrm{X}$ & $\mathrm{X}$ & & & & & & $\begin{array}{l}\text { SAV F-4114 (S) } \\
\text { SAV F-4119 (H) }\end{array}$ \\
\hline Total & 215 & 121 & 126 & 72 & 74 & 80 & 75 & 33 & \\
\hline
\end{tabular}

Tab. 4. List of all fungal species on other substrates recorded at investigated sites.

General abbreviations: N - taxon new to Slovakia, R - rare, interesting or potentially endangered species. Abbreviations of sites: B - Badínsky prales, D - Dobročský prales, H - Havešová, K - Klenovský Vepor, O - Oblík, S - Stužica, V - Vihorlat (for details, see Material and methods). 
ADAMČÍK S. ET AL.: FUNGI AND LICHENS OF NATURAL BEECH FORESTS IN SLOVAKIA

\begin{tabular}{|c|c|c|c|c|c|c|c|c|c|c|}
\hline Status & Species & $\mathbf{H}$ & $\mathbf{S}$ & $\mathbf{V}$ & $\mathbf{0}$ & $\mathbf{K}$ & D & B & Substrate & Specimen(s) \\
\hline $\mathrm{R}$ & $\begin{array}{l}\text { Amylocystis lapponica (Romell) } \\
\text { Bondartsev \& Singer ex Singer }\end{array}$ & & & & & $\mathrm{X}$ & $\mathrm{X}$ & & Picea & $\begin{array}{l}\text { SAV F-3413 (D) } \\
\text { SAV F-3462 (K) }\end{array}$ \\
\hline \multirow[t]{2}{*}{$\mathrm{N}$} & Asterostroma medium Bres. & & $\mathrm{X}$ & & & & & & Abies & PRM 899409 (S) \\
\hline & $\begin{array}{l}\text { Bondarzewia mesenterica (Schaeff.) } \\
\text { Kreisel }\end{array}$ & & & & & & $\mathrm{X}$ & & Abies & SAV F-3409 (D) \\
\hline $\mathrm{R}$ & $\begin{array}{l}\text { Chrysomphalina grossula (Pers.) } \\
\text { Norvell, Redhead \& Ammirati }\end{array}$ & & & & & & $\mathrm{X}$ & & Abies? & SL0 F-1311 (D) \\
\hline $\mathrm{R}$ & Clitocybula abundans (Peck) Singer & & & & & & $\mathrm{X}$ & & Abies & PRM 899420 (D) \\
\hline \multirow[t]{4}{*}{$\mathrm{R}$} & Clitocybula familia (Peck) Singer & & $\mathrm{X}$ & & & & $\mathrm{X}$ & & Abies & $\begin{array}{c}\text { SAV F-3470 (S) } \\
\text { PRM } 899421 \text { (D) }\end{array}$ \\
\hline & $\begin{array}{l}\text { Contumyces rosellus (M.M. Moser) } \\
\text { Redhead, Moncalvo, Vilgalys \& Lutzoni }\end{array}$ & & & & $\mathrm{X}$ & $\mathrm{X}$ & & & $\begin{array}{c}\text { unidentified } \\
\text { fungus }\end{array}$ & $\begin{array}{l}\text { PSG } 4713(0) \\
\text { SAV F-4130 (K) }\end{array}$ \\
\hline & Cosmospora coccinea Rabenh. & & & & $\mathrm{X}$ & & & & $\begin{array}{l}\text { Inonotus } \\
\text { nodulosus }\end{array}$ & PSG $4257(0)$ \\
\hline & Dendrothele acerina (Pers.) P.A. Lemke & & & $\mathrm{X}$ & & & & & Acer & \\
\hline \multirow[t]{3}{*}{$\mathrm{N}$} & $\begin{array}{l}\text { Entoloma hispidulum (M. Lange) } \\
\text { Noordel. }\end{array}$ & & & & & & $\mathrm{X}$ & & Acer & SAV F-3456 (D) \\
\hline & $\begin{array}{l}\text { Fomitopsis rosea (Alb. \& Schwein.) } \\
\text { P. Karst. }\end{array}$ & & & & & & $\mathrm{X}$ & & Picea & SAV F-3472 (D) \\
\hline & $\begin{array}{l}\text { Gloeophyllum odoratum (Wulfen) } \\
\text { Imazeki }\end{array}$ & & & & & & & $\mathrm{X}$ & Picea? & \\
\hline $\mathrm{R}$ & Gymnopilus bellulus (Peck) Murrill & & $\mathrm{X}$ & & & & & & Abies & PRM $899411(\mathrm{~S})$ \\
\hline \multirow[t]{2}{*}{$\mathrm{R}$} & $\begin{array}{l}\text { Hemistropharia albocrenulata (Peck) } \\
\text { Jacobsson \& E. Larss. }\end{array}$ & & & & & & $\mathrm{X}$ & & Picea & PRM 899422 (D) \\
\hline & Hericium flagellum (Scop.) Pers. & & & & & $\mathrm{X}$ & & & Abies & \\
\hline $\mathrm{R}$ & $\begin{array}{l}\text { Hydropus atramentosus (Kalchbr.) } \\
\text { Kotl. \& Pouzar }\end{array}$ & & $\mathrm{X}$ & & & & & & Abies & PVKU 244 (S) \\
\hline \multirow[t]{9}{*}{$\mathrm{N}$} & $\begin{array}{l}\text { Hymenochaete ulmicola } \\
\text { Corfixen \& Parmasto }\end{array}$ & & & & & & & $\mathrm{X}$ & Ulmus & SAV F-3432 (B) \\
\hline & Hypomyces sp. & & $\mathrm{X}$ & & & & & & $\begin{array}{c}\text { unidentified } \\
\text { fungus }\end{array}$ & \\
\hline & Phellinus chrysoloma (Fr.) Donk & & & & & & $\mathrm{X}$ & & Picea? & \\
\hline & $\begin{array}{l}\text { Phellinus hartigii (Allesch. \& Schnabl) } \\
\text { Pat. }\end{array}$ & & & & & & $\mathrm{X}$ & & Abies & \\
\hline & Pholiota flammans (Batsch) P. Kumm. & & $\mathrm{X}$ & & & & & & Abies & SL0 F-1271 (S) \\
\hline & Pholiota squarrosa (Weigel) P. Kumm. & & & & & & $\mathrm{X}$ & & Abies & SAV F-3457 (D) \\
\hline & Pleurocybella porrigens (Pers.) Singer & & $\mathrm{X}$ & & & & & & Abies & PRM $899410(\mathrm{~S})$ \\
\hline & Pycnoporellus fulgens (Fr.) Donk & & & & $\mathrm{X}$ & & $\mathrm{X}$ & & Abies? & SAV F-3453 (D) \\
\hline & $\begin{array}{l}\text { Scytinostroma portentosum } \\
\text { (Berk. \& M.A. Curtis) Donk }\end{array}$ & & & $\mathrm{X}$ & & & & & Salix & SAV F-4129 (V) \\
\hline \multirow[t]{2}{*}{$\mathrm{R}$} & Tricholomopsis osiliensis Vauras & & & & & & & $\mathrm{X}$ & Abies & PRM 899423 (B) \\
\hline & Xylaria longipes Nitschke & & & $\mathrm{X}$ & & & & & Acer? & SL0 F-1304 (V) \\
\hline Total & 27 & 0 & 7 & 3 & 3 & 3 & 12 & 3 & & \\
\hline
\end{tabular}


Tab. 5. Site-specific species represented by triplets of species with highest and lowest $\Delta$ frequency (difference between frequency of the species at a particular site and average frequency of all visited sites). Frequency of species at a site is defined as percentage of positively scored beech logs.

\begin{tabular}{|l|l|l|}
\hline Visited sites & high $\Delta$ frequency & low $\Delta$ frequency \\
\hline Havešová & $16.4 \%$ Ceriporiopsis gilvescens & $-24.0 \%$ Fomes fomentarius \\
& $14.9 \%$ Ischnoderma resinosum & $-14.6 \%$ Mycena galericulata \\
& $13.3 \%$ Trichaptum biforme & $-9.1 \%$ Stereum hirsutum \\
\hline Stužica & $13.6 \%$ Rigidoporus sanguinolentus & $-16.1 \%$ Eutypa spinosa \\
& $11.9 \%$ Panellus stipticus & $-11.9 \%$ Pleurotus pulmonarius \\
& $11.4 \%$ Kretzschmaria deusta & $-9.3 \%$ Pluteus cervinus \\
\hline Vihorlat & $28.7 \%$ Mycena galericulata & $-18.1 \%$ Annulohypoxylon cohaerens \\
& $7.5 \%$ Bisporella citrina & $-16.1 \%$ Eutypa spinosa \\
& $6.3 \%$ Phlebia rufa & $-15.2 \%$ Pleurotus pulmonarius \\
\hline Oblík & $20.0 \%$ Trichaptum biforme & $-16.3 \%$ Mycena galericulata \\
& $16.9 \%$ Annulohypoxylon cohaerens & $-13.0 \%$ Ganoderma applanatum \\
& $9.7 \%$ Mycetinis alliaceus & $-12.4 \%$ Oudemansiella mucida \\
\hline Klenovský Vepor & $16.6 \%$ Oudemansiella mucida & $-16.0 \%$ Kretzschmaria deusta \\
& $14.8 \%$ Polyporus varius & $-11.0 \%$ Mycena crocata \\
& $14.2 \%$ Mycena galericulata & $-10.2 \%$ Hypoxylon fragiforme \\
\hline Dobročský prales & $21.6 \%$ Pleurotus pulmonarius & $-10.8 \%$ Mycena galericulata \\
& $20.3 \%$ Bjerkandera adusta & $-10.0 \%$ Trichaptum biforme \\
& $20.3 \%$ Fomes fomentarius & $-7.7 \%$ Mycena arcangeliana \\
\hline
\end{tabular}

\section{LICHENS}

\section{Summary of results}

In total, 128 lichens and lichenicolous fungi of 69 genera were recorded on bark or wood of living or dead beech trunks in the seven investigated National Nature Reserves (Tab. 6). One lichen, Alyxoria ochrocheila, is listed for the first time in Slovakia. In addition, 28 records of 26 species from other substrates are reported and discussed below (Tab. 7). Twelve species were recorded on beech trunks at all seven sites, half of them with a frequency of more than $50 \%$ at one site or more: Graphis scripta, Lepraria lobificans, Melanelixia glabratula, Phlyctis argena, Pyrenula nitida, Violella fucata. Six additional species were recorded at all seven sites but always with lower frequency/cover: Alyxoria varia, Coenogonium pineti, Lecanora chlarotera, Lecanora glabrata, Lecanora pulicaris, Lepraria incana, while three species were recorded at six sites and showed high frequencies (> 50\%) at one site or more: Cladonia coniocraea, Hypogymnia physodes, Pseudosagedia aenea.

The number of recorded lichen and lichenicolous species per site was very variable (Fig. 4). The highest number was recorded at Stužica (79 species), Klenovský Vepor (69 species) and Havešová (52 species). The low number of species recorded at Badínsky prales reflect the short visit at this site, allowing only 




Fig. 4. Comparison of lichen diversity and presence of red-listed and indicator species on beech trees at investigated sites.

six beech trees to be investigated. The relatively high average of species recorded per tree (8 species) suggests that this number would have been much higher if more trees had been investigated.

The average number of species investigated per tree ranged from 5 to 8 at all sites except of Stužica, where 10 species were recorded on average. Almost half of the species were recorded only at a single site (50 species, 39.4\%) and another 27 species (21.3\%) at two sites only. This suggests a high amount of site-specific lichen and lichenicolous species.

\section{Red-listed species}

Almost half (60 species) of all the epiphytic lichens recorded on beech trees appeared in the Red list of Slovakia (Pišút et al. 2001). Of these, 2 species were classified as extinct (EX), 22 as critically endangered (CR), 10 as endangered (EN), 15 as vulnerable (VU), and 9 as near threatened (NT), while 2 were classified in the data deficient (DD) category. The highest number and proportion of red-listed species was recorded at Stužica (38 species, $48 \%$ of all species re- 
corded at the site) and Klenovský Vepor (30 species, 43\% of all species recorded at the site). A relatively high number of red-listed species was also recorded from Havešová (23 species, $44 \%$ of all species recorded at the site). These three sites also showed the highest number of CR species, 14 at Stužica, 10 at Klenovský Vepor and 10 at Havešová.

Alyxoria ochrocheila, collected at Klenovský Vepor, is reported from Slovakia for the first time. It is widespread and well known from West Europe and Canada, but only few reports are from Central and East Europe (Suija et al. 2010 from Estonia and Bielczyk et al. 2004 from Poland).

Other important records include Eopyrenula leucoplaca and Lecanographa amylacea recorded at Havešová. Both species have been regarded as extinct from Slovakia for decades. Eopyrenula leucoplaca grows on rough bark of deciduous trees. It is more common in Scandinavia but rare and declining in South and Central Europe (Aptroot 1991). The previous Slovak reports were from the surroundings of the cities of Prešov and Bratislava in the $19^{\text {th }}$ century (e.g. Hazslinszky 1884, Zahlbruckner 1894), but it was recently reported from Muránska planina National Park by Malíček et al. (2014). Lecanographa amylacea has a predominantly Subatlantic distribution in Europe (Wirth et al. 2013); it is mostly found growing on bark of veteran oak trees in open light conditions. This species had not been reported from Slovakia since the $19^{\text {th }}$ century (Hazslinszky 1884; eastern part of the country: Prešov, Košice, Lipovce).

Among the 22 critically endangered lichens recorded, several deserve extra attention. The very rare crustose lichen Belonia herculina (recorded in Havešová and Stužica) is known from beech trunks (but also Acer pseudoplatanus) in oldgrowth beech or mixed forests in Slovakia (Pišút et al. 2007). It was considered an endemic to the Carpathians for a long time, but was recently reported from the Slovenian Alps (Arup et al. 2001), Crimean mountains (Khodosovtsev et al. 2013) and the Caucasus (Urbanavichyus 2010). In the last century, the species showed a sharp decline in Slovakia (Lackovičová et al. 2001), and during the last twenty years it had only been reported from the Poloniny Mts. and the Strážovské vrchy Mts. (Pišút et al. 2007, Guttová \& Pišút 2004). Gyalecta flotowii and G. truncigena were both known from scattered localities across Slovakia in the first half of the last century. During the last two decades reports have been very scarce. Gyalecta flotowii is recently only known from Stužica (Pišút et al. 2007, Vondrák et al. 2015) and in this paper Havešová is reported as a new locality. Gyalecta truncigena was considered to be extinct for a long time, but recently it was reported from the Muránska planina National Park and Stužica (Guttová \& Palice 2004, Vondrák et al. 2015). Our collections from Havešová and Oblík represent new localities in Slovakia. Thelopsis rubella was first reported from Slovakia by Zahlbruckner (1894) from the Malé Karpaty Mts. (W Slovakia), then by Suza (1923) from the Strážovské vrchy Mts. For nearly 50 years it had been considered 
to be extinct from Slovakia, until it was reported from the Belianske Tatry Mts. (Pišút 1986) and Muránska planina Mts. (Guttová \& Palice 2004). Our collections from Havešová and Dobročský prales represent new localities for the country. Thelotrema lepadinum is globally a widespread species (from the tropical to the temperate belt) growing in humid climates on trees with smooth bark. It is very sensitive to air pollution and rare in Slovakia (Pišút \& Lackovičová 1992). It was recorded at three of the investigated sites (Badínsky prales, Dobročský prales and Stužica).

Some crustose lichens collected during our research are relatively frequent in old-growth beech forests but are sensitive to air pollution and declining in industrial and urban areas, e.g. Bacidia rubella, Graphis scripta, Pyrenula nitida, Strigula stigmatella, etc. Arthonia didyma, a species associated with beech trunks in submontane forests from Scandinavia to Northern Spain and Italy (Nimis 1993), has recently been reported only from the Belianske Tatry Mts., Muránska planina Mts. and Stužica (Lisická 2005, Guttová \& Palice 2004, Vondrák et al. 2015). Our reports from Dobročský prales, Havešová and Klenovský Vepor represent important contributions to the knowledge of the distribution of probably overlooked species in Slovakia.

Lobaria pulmonaria shows a Suboceanic-montane distribution in Europe. This, in Slovakia, critically endangered epiphyte is restricted to ancient beech and mixed forests with high air humidity. It is very sensitive to air pollution and has disappeared from many parts of Central Europe in the late $20^{\text {th }}$ century. The distribution of Lobaria pulmonaria in Slovakia is well documented. It was widely distributed on the whole territory at the beginning of the last century, but is recently known from less than 50 localities (Pišút \& Liška 1995, Lackovičová et al. 2001).

\section{Indicator species}

Index of ecological continuity (IEC) reflecting human influence on sites was evaluated by counting the number of indicator species following Pišút (1997). Between 6 and 19 indicator species were reported from the seven investigated sites which could be classified in three categories (number of indicators per site in parenthesis): high quality - Stužica (19), Klenovský Vepor (18), Havešová (15); good quality - Dobročský prales (12); weak quality - Oblík (9), Vihorlat (8), Badínsky prales (6). Including data from other phorophytes (trees) would probably increase the IEC to even higher values, incorporating the category 'outstanding'.

\section{Rare species and interesting records}

In addition, we recorded some species of epiphytic lichens considered to be rare in Slovakia but not included in the national Red list (Pišút et al. 2001). Arthonia helvola, recorded at Havešová, Vihorlat and Oblík, was previously 
known only from Muránska planina National Park (Guttová \& Palice 1999) and Stužica (Vondrák et al. 2015). The distribution of this species in Europe is disjunctive and insufficiently known. In the Czech Republic, it was recorded from deciduous forests close to industrial areas, so the species seems to be relatively toxitolerant (Palice 1999). Agonimia allobata is another species only known from the Muránska planina National Park (Guttová \& Palice 1999, Guttová \& Palice 2002) and Stužica (bark of Acer pseudoplatanus, Vondrák et al. 2015). We recorded it on beech at Badínsky prales, Dobročský prales, Havešová and Oblík. Anisomeridium polypori is a cosmopolitan species which we recorded on beech trunks at all investigated sites except Klenovský Vepor. In Slovakia it was previously reported only from Muránska planina National Park (bark of Acer pseudoplatanus; Guttová \& Palice 2002) and Stužica (also on Ulmus, Fagus; Vondrák et al. 2015). Our records from six of the seven investigated sites suggest that it is often overlooked.

\section{Interesting collections from other substrates}

All 26 lichen species recorded on wood or bark of other tree species and on rocks are listed in the Tab. 7. Most of them are red-listed (16 species) and/or indicator species (13 species). Gyalecta ulmi recorded on bark of Acer platanoides at Dobročský prales is currently very rare and declining in Europe. In Slovakia, it was known from 21 major geographical units (Lukniš \& Mazúr 1980) before 1989, but after 1990 it was reported only from six of them (Lackovičová \& Guttová 2006). An important find for the country is Ramalina carpatica (on rock at Klenovský Vepor) that has been usually recorded from higher altitudes (Lisická 2005).

\section{Comparison of our recent results with historical data}

Old-growth forests usually have a wide range of substrates, suitable light conditions and relatively high air humidity, favouring a high diversity of rare and endangered epiphytic lichen species (Guttová \& Lackovičová 2011). Our results not only contributed to the knowledge of distribution of rare lichens, but also represent a significant contribution to the knowledge of the species diversity of the studied sites and corresponding regions.

Havešová was poorly known prior to our study. During short visits to the site in 1993 and 1996, I. Pišút, A. Lackovičová and A. Guttová collected only 15 species on beech trunks, e.g. Buellia schaereri, Cetrelia cetrarioides, Lecania fuscella, Parmotrema perlatum, Pertusaria pertusa, P. nitidella (Guttová 1997, Pišút \& Guttová 1997). In the current study, we report 52 species recorded on beech trunks (7 species per tree on average), 47 of them new to Havešová. Almost half of these (23 species) are red-listed (Pišút et al. 2001). Two red-listed 
species, Eopyrenula leucoplaca and Lecanographa amylacea, had been treated as extinct (EX). Ten red-listed species are classified as endangered (EN), eight as vulnerable (VU), three as near threatened (NT) and one in the data deficient (DD) category. The number of 15 indicator species demonstrates the relatively high natural value of this site.

Stužica is known as one of the most important lichenological sites in Slovakia. The first investigations by I. Pišút and J. Liška in the 1960s and 1970s provided information on the occurrence of e.g. Leptogium cyanescens, Lobaria pulmonaria, Menegazzia terebrata, Parmeliella triptophylla and Thelotrema lepadinum (Pišút 1963). Pišút \& Lackovičová (1992) and Pišút et al. (2007) published a study on lichens in the Bukovské vrchy Mts. (incl. Stužica). The most comprehensive study is by Vondrák et al. (2015), reporting 230 epiphytic and epixylic lichens from Stužica, which is the highest number known for this type of Central European forest (Vondrák et al. 2015). Among them, 95 species were previously not reported from beech trunks and, together with historical data, 186 species on beech trunks were reported from Stužica prior to our study. Seven species out of the 79 reported from Stužica in this study are new to the area, among them some rare or uncommon ones (e.g. Gyalecta truncigena), while others are common oligotrophic species (e.g. Cladonia coniocraea, Coenogonium pineti and Imshaugia aleurites). In addition, 11 species recorded on beech trunks during this study were previously reported only from other trees, mainly from Acer and Abies (e.g. Calicium salicinum, Lecanora expallens, Micarea peliocarpa, Normandina pulchella, Opegrapha vulgata). More than $48 \%$ of the lichens from Stužica are red-listed: 14 CR, 5 EN, 12 VU and 7 NT. With 19 species, indicators of ecological continuity are also well represented.

Vihorlat is a National Nature Reserve situated in Vihorlatské vrchy Mts. Reports by F. Hazslinszky and H. Lojka from the $19^{\text {th }}$ century (e.g. Hazslinszky 1862) are among the oldest Slovak lichenological publications. They were followed by investigations by Szatala (1916, 1923), Servít \& Nádvorník (1932), Pišút (1985, 1987), Lackovičová et al. (2004) and others. In total, more than 160 epiphytic and epixylic lichen species have been reported from the Vihorlatské vrchy Mts., among them the rare Oceanic species Nephroma bellum, N. parille, Heterodermia speciosa and Pyrenula laevigata. The last two have not been found recently. They are now considered to be extinct. The same goes for Stictis urceolatum, which was known from a single site in this area and has never been reported from neighbouring countries (Pišút 1987). Previously, only 22 epiphytic species (incl. 10 on beech) were recorded from Vihorlat National Nature Reserve. There is however confusion in the naming of the sites. Data published by Lackovičová et al. (2004) from Vihorlatský prales do not originate from the site reported here, but from Kyjovský prales National Nature Reserve (in the Vihorlatské vrchy Mts.). Among the 31 species detected by the Beech Boys, 28 are reported from 
Vihorlat in this study for the first time. Thirteen species are red-listed ( $3 \mathrm{CR}, 4 \mathrm{EN}$, $2 \mathrm{VU}, 3 \mathrm{NT}, 1 \mathrm{DD})$. Occurrence of some rare species considered as indicators of clean air and reported from the site in the past (e.g. Menegazzia terebrata, Lobaria pulmonaria and Leptogium saturninum) was not confirmed. The decline of these species in Slovakia is caused by an increase in air pollution. For example, Leptogium saturninum, known from nearly all Slovak mountains, has been reported from only four sites in Slovakia since 1960 (Pišút \& Liška 1995).

Oblík is situated in the Slanské vrchy Mts., an area investigated in the past by F. Hazslinszky, H. Lojka, J. Suza, I. Pišút and J. Liška (Pišút \& Liška 1985). A total of 170 epiphytic and epixylic lichens out of the 380 lichen species are known from the Slanské vrchy Mts. So far only eleven species have been reported from Oblík (with only Phlyctis argena recorded on beech). Of the 44 species reported from Oblík in this study (average 5 per tree), 32 are new to the area. Fifteen of them are red-listed (4 CR, 4 EN, 3 VU, 3 NT, 1 DD) and nine are indicator species.

Dobročský prales is a relatively well-known natural beech forest in Slovakia, but the first lichen report (Usnea filipendula) is quite recent (Križová 2002). Lackovičová \& Pišút (2004) reported 62 lichen species from the reserve and its protection zone. One of the reported species by Lackovičová \& Pišút (2004) was Lecanora leuckertiana, which is a misidentification according to a revision of the corresponding herbarium material (deposited in SAV) by M. Kukwa (Kukwa, pers. comm.). The correct identity of this lichen is Lepraria ecorticata (L.R. Laundon) Kukwa. This information has not been published previously, so this is the first time it is reported from Slovakia. In this study, 39 epiphytic lichens (average 7 per tree) are reported from Dobročský prales. Fifteen of them are red-listed in Slovakia (6 CR, 4 EN, 4 VU, 1 NT), twelve are indicator species.

Badínsky prales was a lichenologically unexplored forest. No lichens had been reported prior to our research except for a single collection of the lichenised basidiomycete Multiclavula mucida (Pišút 2000). Our brief visit (only 6 investigated trunks) resulted in the collection of 22 epiphytic lichens on beech (average 8 per tree), all of them new to the site. Seven species are red-listed (2 $\mathrm{CR}, 3 \mathrm{EN}, 2 \mathrm{VU}$ ), and six species are indicators of ecological continuity of natural montane forests in Slovakia (Pišút 1997).

Also Klenovský Vepor National Nature Reserve did not have any historical lichen reports. It is situated on a hilltop only ca. $5 \mathrm{~km}$ east of Dobročský prales. Only few lichens (Evernia divaricata, Usnea dasypoga, U. florida, U. glabrescens and Peltigera degenii) are known from neighbouring northern slopes (Lackovičová \& Pišút 2004). This study revealed 69 lichens on beech (average 7 per tree). Because of the lack of published data, they all represent new records for Klenovský Vepor. Alyxoria ochrocheila is new to Slovakia. A total of 30 red-listed species (10 CR, $7 \mathrm{EN}, 7 \mathrm{VU}, 5 \mathrm{NT}, 1 \mathrm{DD})$ and 18 indicator species confirm the importance and natural value of the site. 
Tab. 6. List of all lichen and lichenicolous species on beech trunks recorded at investigated sites incl. frequencies (as percentage of occurrence on investigated trunks).

General abbreviations: N - taxon new to Slovakia, F - non-lichenised fungi, I - indicator species of conservation value of beech forests, $\mathrm{RL}$ - red-listed species, $\mathrm{X}$ - additional records from non-stratified sampling.

Threatened species categories: EX - extinct, CR - critically endangered, EN - endangered, VU - vulnerable, NT - near threatened, DD - data deficient.

Abbreviations of investigated sites: B - Badínsky prales, D - Dobročský prales, H - Havešová, K Klenovský Vepor, O - Oblík, S - Stužica, V - Vihorlat (for details, see Material and methods).

Species represented in herbarium SAV and their origin are indicated in the last column.

\begin{tabular}{|c|c|c|c|c|c|c|c|c|c|c|}
\hline Status & Species & RL & H & $\mathbf{S}$ & V & $\mathbf{0}$ & $\mathbf{K}$ & D & B & SAV \\
\hline \multirow[t]{3}{*}{$\mathrm{I}$} & Acrocordia gemmata (Ach.) A. Massal. & & $10 \%$ & & & $4 \%$ & & & & \\
\hline & Agonimia allobata (Stizenb.) P. James & & $10 \%$ & & & $9 \%$ & & $15 \%$ & $\mathrm{X}$ & $\mathrm{H}, \mathrm{O}, \mathrm{D}$ \\
\hline & Agonimia tristicula (Nyl.) Zahlbr. & DD & & & & & $4 \%$ & & & \\
\hline $\mathrm{N}$ & Alyxoria ochrocheila (Nyl.) Ertz \& Tehler & & & & & & $4 \%$ & & & $\mathrm{~K}$ \\
\hline \multirow[t]{3}{*}{$\mathrm{I}$} & Alyxoria varia (Pers.) Ertz \& Tehler & $\mathrm{VU}$ & $10 \%$ & $8 \%$ & $4 \%$ & $4 \%$ & $4 \%$ & $15 \%$ & $\mathrm{X}$ & \\
\hline & $\begin{array}{l}\text { Amandinea punctata (Hoffm.) } \\
\text { Coppins \& Scheid. }\end{array}$ & & & $8 \%$ & & $13 \%$ & & & & S \\
\hline & $\begin{array}{l}\text { Anisomeridium polypori (Ellis \& Everh.) } \\
\text { M.E. Barr }\end{array}$ & & $10 \%$ & $12 \%$ & $8 \%$ & $4 \%$ & & $4 \%$ & $\mathrm{X}$ & $0, \mathrm{D}$ \\
\hline $\mathrm{I}$ & Arthonia didyma Körb. & $\mathrm{CR}$ & $5 \%$ & & & & $12 \%$ & $4 \%$ & & $\mathrm{~K}$ \\
\hline I & Arthonia helvola (Nyl.) Nyl. & DD & $10 \%$ & & $21 \%$ & $9 \%$ & & & & $\mathrm{H}, \mathrm{V}$ \\
\hline I & Arthonia radiata (Pers.) Ach. & $\mathrm{CR}$ & $10 \%$ & $4 \%$ & & $13 \%$ & $12 \%$ & & & \\
\hline I & Arthonia ruana A. Massal. & $\mathrm{EN}$ & $14 \%$ & & & $4 \%$ & & $4 \%$ & & \\
\hline \multirow[t]{10}{*}{ I } & Arthonia spadicea Leight. & & & & & & $4 \%$ & $11 \%$ & & $\mathrm{D}$ \\
\hline & Athallia holocarpa Arup, Frödén \& Søchting & & & & & & $4 \%$ & & & \\
\hline & $\begin{array}{l}\text { Athallia cf. pyracea (Ach.) } \\
\text { Arup, Frödén \& Søchting }\end{array}$ & & & & & & $4 \%$ & & & \\
\hline & Bacidia circumspecta (Nyl. ex Vain.) Malme & & & & & $4 \%$ & & & & 0 \\
\hline & Bacidia incompta (Borrer ex Hook.) Anzi & & & & & & & $\mathrm{X}$ & & $\mathrm{D}$ \\
\hline & Bacidia rubella (Hoffm.) A. Massal. & $\mathrm{VU}$ & $10 \%$ & $8 \%$ & & $4 \%$ & $12 \%$ & $11 \%$ & & $\mathrm{D}$ \\
\hline & Bacidia subincompta (Nyl.) Arnold & & & & & $4 \%$ & & $4 \%$ & & $0, \mathrm{D}$ \\
\hline & Bacidina arnoldiana (Körb.) V. Wirth \& Vězda & & & & & $4 \%$ & & & & \\
\hline & $\begin{array}{l}\text { Bacidina delicata (Larbal. ex Leight.) } \\
\text { V. Wirth \& Vězda }\end{array}$ & & $\mathrm{X}$ & & & & & & & \\
\hline & Bacidina phacodes (Körb.) Vẽzda & & $10 \%$ & & & & $4 \%$ & $15 \%$ & & $\mathrm{H}, \mathrm{D}$ \\
\hline \multirow[t]{8}{*}{ I } & Belonia herculina (Rehm ex Lojka) Hazsl. & $\mathrm{CR}$ & $14 \%$ & $4 \%$ & & & & & & $\mathrm{H}, \mathrm{S}$ \\
\hline & Biatora efflorescens (Hedl.) Räsänen & $\mathrm{VU}$ & $19 \%$ & $40 \%$ & & $4 \%$ & & & & \\
\hline & Biatora globulosa (Flörke) Fr. & & $5 \%$ & $4 \%$ & & $4 \%$ & & & & \\
\hline & Biatora ocelliformis (Nyl.) Arnold & & & & & & & $4 \%$ & & $\mathrm{D}$ \\
\hline & Biatora vernalis (L.) Fr. & & & $4 \%$ & & & & & & $\mathrm{~S}$ \\
\hline & Biatoridium monasteriense J. Lahm & $\mathrm{CR}$ & $5 \%$ & $4 \%$ & & & $4 \%$ & $7 \%$ & & $\mathrm{D}$ \\
\hline & Bilimbia sabuletorum (Schreb.) Arnold & & & $4 \%$ & & & & & & \\
\hline & $\begin{array}{l}\text { Buellia griseovirens } \\
\text { (Turner \& Borrer ex Sm.) Almb. }\end{array}$ & & & $12 \%$ & & $4 \%$ & $4 \%$ & & & S \\
\hline
\end{tabular}


CZech Mycology 68(1): 1-40, February 1, 2016 (ONLINE VERSION, ISSN 1805-1421)

\begin{tabular}{|c|c|c|c|c|c|c|c|c|c|c|}
\hline Status & Species & RL & $\mathbf{H}$ & $\mathbf{S}$ & $\mathbf{V}$ & $\mathbf{0}$ & $\mathbf{K}$ & D & B & SAV \\
\hline $\mathrm{I}$ & Calicium glaucellum Ach. & $\mathrm{CR}$ & & & & & $8 \%$ & & & $\mathrm{~K}$ \\
\hline \multirow[t]{5}{*}{ I } & Calicium salicinum Pers. & EN & & $4 \%$ & $8 \%$ & & $4 \%$ & & & $\mathrm{~V}, \mathrm{~K}$ \\
\hline & Caloplaca holocarpa (Ach.) Wade & & & & & & $4 \%$ & & & \\
\hline & Candelariella efflorescens auct. europ. & & & $4 \%$ & & & $4 \%$ & & & $\mathrm{~K}$ \\
\hline & Candelariella xanthostigma (Ach.) Lettau & & & $12 \%$ & & & $8 \%$ & & & $\mathrm{~K}$ \\
\hline & $\begin{array}{l}\text { Cetrelia olivetorum (Nyl.) } \\
\text { W.L. Culb. \& C.F. Culb., sensu lato } \\
\end{array}$ & $\mathrm{VU}$ & & $32 \%$ & & & $4 \%$ & & & $\mathrm{~S}$ \\
\hline I & Chaenotheca brachypoda (Ach.) Tibell & $\mathrm{CR}$ & & & $4 \%$ & & & & & $\mathrm{~V}$ \\
\hline $\mathrm{I}$ & Chaenotheca chlorella (Ach.) Müll. Arg. & $\mathrm{EN}$ & & & $4 \%$ & & & & & $\mathrm{~V}$ \\
\hline I & $\begin{array}{l}\text { Chaenotheca chrysocephala } \\
\text { (Turner ex Ach.) Th. Fr. }\end{array}$ & $\mathrm{VU}$ & & & & & $\mathrm{X}$ & & & K \\
\hline I & Chaenotheca cf. stemonea (Ach.) Müll. Arg. & $\mathrm{CR}$ & & & $4 \%$ & & & & & $\mathrm{~V}$ \\
\hline I & Chaenotheca trichialis (Ach.) Th. Fr. & $\mathrm{CR}$ & & & & & $8 \%$ & $4 \%$ & & $\mathrm{~K}, \mathrm{D}$ \\
\hline $\mathrm{F}$ & $\begin{array}{l}\text { Chaenothecopsis consociata (Nádv.) } \\
\text { A.F.W. Schmidt }\end{array}$ & & & & & & $\mathrm{X}$ & & & \\
\hline $\mathrm{F}$ & Chaenothecopsis pusilla (Ach.) A.F.W. Schmidt & & $5 \%$ & $4 \%$ & & $4 \%$ & $4 \%$ & & & \\
\hline \multirow[t]{5}{*}{$\mathrm{F}$} & $\begin{array}{l}\text { Chaenothecopsis cf. reagens (Nádv.) } \\
\text { A.F.W. Schmidt }\end{array}$ & & $5 \%$ & & & & & & & \\
\hline & Cladonia coniocraea (Flörke) Spreng. & & $43 \%$ & $60 \%$ & $50 \%$ & $26 \%$ & & $4 \%$ & $\mathrm{X}$ & \\
\hline & Cladonia digitata (L.) Hoffm. & & & $4 \%$ & & & $12 \%$ & & & \\
\hline & Cladonia fimbriata (L.) Fr. & & $10 \%$ & $4 \%$ & & & & & & \\
\hline & $\begin{array}{l}\text { Coenogonium pineti (Schrad. ex Ach.) } \\
\text { Lücking \& Lumbsch }\end{array}$ & & $38 \%$ & $8 \%$ & $25 \%$ & $30 \%$ & $8 \%$ & $7 \%$ & $\mathrm{X}$ & \\
\hline \multirow[t]{3}{*}{$\mathrm{I}$} & Collema flaccidum (Ach.) Ach. & EN & $5 \%$ & & & & $4 \%$ & & & $\mathrm{H}$ \\
\hline & Eopyrenula leucoplaca (Wallr.) R.C. Harris & EX & $5 \%$ & & & & & & & \\
\hline & Evernia prunastri (L.) Ach. & EN & $5 \%$ & $4 \%$ & & $4 \%$ & $4 \%$ & & & \\
\hline \multirow[t]{2}{*}{ I } & Fuscidea cyathoides (Ach.) V. Wirth \& Vẽzda & $\mathrm{CR}$ & & $4 \%$ & & & & & & $\mathrm{~S}$ \\
\hline & Fuscidea sp. & & $5 \%$ & $4 \%$ & & $13 \%$ & $27 \%$ & $4 \%$ & & \\
\hline $\mathrm{I}$ & Graphis scripta (L.) Ach. & $\mathrm{EN}$ & $86 \%$ & $36 \%$ & $38 \%$ & $26 \%$ & $23 \%$ & $63 \%$ & $\mathrm{X}$ & $\mathrm{H}, \mathrm{S}, \mathrm{V}$ \\
\hline \multirow[t]{3}{*}{ I } & Gyalecta flotowii Körb. & $\mathrm{CR}$ & $14 \%$ & $4 \%$ & & & & & & $\mathrm{H}$ \\
\hline & Gyalecta truncigena (Ach.) Hepp & $\mathrm{CR}$ & $5 \%$ & $4 \%$ & & $4 \%$ & & & & \\
\hline & $\begin{array}{l}\text { Hypocenomyce scalaris (Ach. ex Lilj.) } \\
\text { M. Choisy }\end{array}$ & & & $4 \%$ & & & & & & \\
\hline \multirow[t]{9}{*}{ I } & Hypogymnia farinacea Zopf & $\mathrm{VU}$ & & & & & $8 \%$ & & & $\mathrm{~K}$ \\
\hline & Hypogymnia physodes (L.) Nyl. & & $5 \%$ & $20 \%$ & $17 \%$ & $4 \%$ & $69 \%$ & $33 \%$ & & \\
\hline & Hypogymnia tubulosa (Schaer.) Hav. & NT & & & & & $4 \%$ & $4 \%$ & & K \\
\hline & Imshaugia aleurites (Ach.) S.L.F. Meyer & $\mathrm{VU}$ & & $8 \%$ & & & & & & \\
\hline & $\begin{array}{l}\text { Lecanographa amylacea (Ehrh. ex Pers.) } \\
\text { Egea \& Torrente }\end{array}$ & $\mathrm{EX}$ & $5 \%$ & & & & & & & $\mathrm{H}$ \\
\hline & Lecanora argentata (Ach.) Malme & & & $24 \%$ & $4 \%$ & & $8 \%$ & & & $\mathrm{~S}, \mathrm{~K}$ \\
\hline & Lecanora carpinea (L.) Vain. & & & $8 \%$ & & & & & & S \\
\hline & Lecanora chlarotera Nyl. & & $19 \%$ & $28 \%$ & $42 \%$ & $48 \%$ & $31 \%$ & $7 \%$ & $\mathrm{X}$ & \\
\hline & Lecanora conizaeoides Nyl. ex Cromb. & & & $4 \%$ & $13 \%$ & & $8 \%$ & & & $\mathrm{~K}$ \\
\hline
\end{tabular}


ADAMČíK S. ET AL.: FUNGI AND LICHENS OF NATURAL BEECH FORESTS IN SLOVAKIA

\begin{tabular}{|c|c|c|c|c|c|c|c|c|c|c|}
\hline Status & Species & RL & $\mathbf{H}$ & $\mathbf{S}$ & V & $\mathbf{0}$ & $\mathbf{K}$ & D & B & SAV \\
\hline & Lecanora expallens Ach. & & & $4 \%$ & & & & & & $\mathrm{~S}$ \\
\hline & Lecanora glabrata (Ach.) Malme & & $43 \%$ & $12 \%$ & $8 \%$ & $9 \%$ & $15 \%$ & $15 \%$ & $\mathrm{X}$ & $\mathrm{K}$ \\
\hline & Lecanora intumescens (Rebent.) Rabenh. & EN & & & & & $4 \%$ & & & $\mathrm{~K}$ \\
\hline & Lecanora pulicaris (Pers.) Ach. & & $5 \%$ & $4 \%$ & $4 \%$ & $4 \%$ & $8 \%$ & $4 \%$ & $\mathrm{X}$ & $\mathrm{V}$ \\
\hline & Lecanora saligna (Schrad.) Zahlbr. & & & $4 \%$ & & & $4 \%$ & & & $\mathrm{~S}, \mathrm{~K}$ \\
\hline & Lecanora subintricata (Nyl.) Th. Fr. & & $5 \%$ & & & & & & & $\mathrm{H}$ \\
\hline & Lecanora varia (Hoffm.) Ach. & VU & & $4 \%$ & & & & & & \\
\hline & Lecidella elaeochroma (Ach.) M. Choisy & & $14 \%$ & & & $13 \%$ & $15 \%$ & & $\mathrm{X}$ & $0, \mathrm{~K}$ \\
\hline & Lecidella scabra (Taylor) Hertel \& Leuckert & & & & & & $4 \%$ & & & \\
\hline & Lepraria incana (L.) Ach. & & $29 \%$ & $36 \%$ & $46 \%$ & $26 \%$ & $12 \%$ & $41 \%$ & $\mathrm{X}$ & \\
\hline & Lepraria lobificans $\mathrm{Nyl}$. & & $57 \%$ & $72 \%$ & $33 \%$ & $35 \%$ & $73 \%$ & $81 \%$ & $\mathrm{X}$ & \\
\hline $\mathrm{I}$ & Leptogium cyanescens (Rabenh.) Körb. & $\mathrm{CR}$ & & $4 \%$ & & & $4 \%$ & & & $\mathrm{H}$ \\
\hline \multirow[t]{2}{*}{ I } & Leptogium lichenoides (L.) Zahlbr. & & & $4 \%$ & & & & & & $\mathrm{H}$ \\
\hline & $\begin{array}{l}\text { Lichenomphalia umbellifera (L.: Fr.) } \\
\text { Redhead, Lutzoni, Moncalvo \& Vilgalys }\end{array}$ & & & $\mathrm{X}$ & & & & & & \\
\hline \multirow[t]{5}{*}{ I } & Lobaria pulmonaria (L.) Hoffm. & $\mathrm{CR}$ & & $12 \%$ & & & $4 \%$ & & & \\
\hline & $\begin{array}{l}\text { Melanelixia glabratula (Lamy) } \\
\text { Sandler \& Arup }\end{array}$ & & $10 \%$ & $40 \%$ & $13 \%$ & $30 \%$ & $27 \%$ & $52 \%$ & $\mathrm{X}$ & $\mathrm{K}$ \\
\hline & Micarea peliocarpa (Anzi) Coppins \& R. Sant. & & & $4 \%$ & & & & & & \\
\hline & Micarea prasina Fr. & & $19 \%$ & & $4 \%$ & $9 \%$ & $8 \%$ & $19 \%$ & & \\
\hline & Multiclavula mucida (Pers.) R.H. Petersen & & $5 \%$ & $\mathrm{X}$ & & & $\mathrm{X}$ & & & $\mathrm{S}$ \\
\hline \multirow[t]{2}{*}{ I } & Normandina pulchella (Borrer) Nyl. & $\mathrm{CR}$ & & $8 \%$ & & & & & & $\mathrm{~S}$ \\
\hline & Ochrolechia androgyna (Hoffm.) Arnold & & & $4 \%$ & & & & & & $\mathrm{~S}$ \\
\hline I & Opegrapha rufescens Pers. & $\mathrm{VU}$ & & & & & $4 \%$ & & & $\mathrm{~K}$ \\
\hline I & $\begin{array}{l}\text { Opegrapha viridis (Pers. ex Ach.) } \\
\text { Behlen \& Desberger }\end{array}$ & $\mathrm{VU}$ & & $4 \%$ & & & & $37 \%$ & $\mathrm{X}$ & $\mathrm{S}, \mathrm{D}$ \\
\hline \multirow[t]{2}{*}{ I } & Opegrapha vulgata Ach., sensu lato & $\mathrm{VU}$ & & $4 \%$ & & & & $4 \%$ & & $\mathrm{D}$ \\
\hline & Parmelia saxatilis (L.) Ach. & NT & & $40 \%$ & $13 \%$ & $4 \%$ & $19 \%$ & & & $\mathrm{~S}$ \\
\hline \multirow[t]{10}{*}{ I } & Parmelia submontana Nádv. ex Hale & $\mathrm{CR}$ & & $4 \%$ & & & & & & $\mathrm{~S}$ \\
\hline & Parmelia sulcata Taylor & & & $48 \%$ & & $4 \%$ & $15 \%$ & $4 \%$ & & \\
\hline & Parmelina tiliacea (Hoffm.) Hale & NT & & $12 \%$ & $4 \%$ & $4 \%$ & $4 \%$ & & & $\mathrm{~V}, 0$ \\
\hline & Parmeliopsis ambigua (Wulfen) Nyl. & & & $24 \%$ & $8 \%$ & $4 \%$ & $8 \%$ & & & $\mathrm{~S}$ \\
\hline & Parmeliopsis hyperopta (Ach.) Arnold & $\mathrm{VU}$ & & $8 \%$ & $8 \%$ & & & & & \\
\hline & $\begin{array}{l}\text { Peltigera praetextata (Flörke ex Sommerf.) } \\
\text { Zopf }\end{array}$ & & $5 \%$ & & & & & $4 \%$ & & \\
\hline & $\begin{array}{l}\text { Pertusaria albescens (Huds.) } \\
\text { M. Choisy \& Werner }\end{array}$ & NT & & $20 \%$ & & & & & & $\mathrm{~S}$ \\
\hline & Pertusaria amara (Ach.) Nyl. & NT & & $44 \%$ & & $4 \%$ & & & & \\
\hline & Pertusaria coccodes (Ach.) Nyl. & $\mathrm{VU}$ & & $4 \%$ & & & & & & $\mathrm{~S}$ \\
\hline & Pertusaria coronata (Ach.) Th. Fr. & $\mathrm{VU}$ & & $4 \%$ & & & & & & $\mathrm{~S}$ \\
\hline \multirow[t]{2}{*}{$\mathrm{I}$} & Pertusaria hymenea (Ach.) Schaer. & $\mathrm{CR}$ & $5 \%$ & & & $13 \%$ & $4 \%$ & $4 \%$ & & $\mathrm{H}$ \\
\hline & Pertusaria pertusa (Weigel) Tuck. & NT & $5 \%$ & & & & & & & \\
\hline I & Phaeophyscia endophoenicea (Harm.) Moberg & $\mathrm{CR}$ & $5 \%$ & $12 \%$ & & & $8 \%$ & & & \\
\hline
\end{tabular}


CZech Mycology 68(1): 1-40, February 1, 2016 (ONLINE VERSion, ISSN 1805-1421)

\begin{tabular}{|c|c|c|c|c|c|c|c|c|c|c|}
\hline Status & Species & $\mathbf{R L}$ & H & $\mathbf{S}$ & V & $\mathbf{0}$ & $\mathbf{K}$ & D & B & SAV \\
\hline & Phaeophyscia orbicularis (Neck.) Moberg & & & $\mathrm{X}$ & & & & & & S \\
\hline & Phlyctis agelaea (Ach.) Flot. & $\mathrm{CR}$ & & & $4 \%$ & & & & & \\
\hline & Phlyctis argena (Spreng.) Flot. & & $38 \%$ & $64 \%$ & $17 \%$ & $9 \%$ & $38 \%$ & $44 \%$ & $\mathrm{X}$ & \\
\hline & Physcia adscendens H. Olivier & & & $4 \%$ & $4 \%$ & & $4 \%$ & & & $\mathrm{~S}, \mathrm{~V}$ \\
\hline & Physcia dubia (Hoffm.) Lettau & & & & & & $8 \%$ & & & $\mathrm{~K}$ \\
\hline & Platismatia glauca (L.) W.L. Culb. \& C.F. Culb. & NT & & $44 \%$ & & & $35 \%$ & & & \\
\hline & Porina leptalea (Durieu \& Mont.) A.L. Sm. & $\mathrm{EN}$ & $19 \%$ & & & & & & $\mathrm{X}$ & $\mathrm{H}$ \\
\hline & Pseudevernia furfuracea (L.) Zopf & NT & & $4 \%$ & $4 \%$ & & $8 \%$ & & & $\mathrm{~K}$ \\
\hline & Pseudosagedia aenea (Wallr.) Zahlbr. & & $38 \%$ & & $4 \%$ & $70 \%$ & $23 \%$ & $11 \%$ & $\mathrm{X}$ & 0 \\
\hline I & Pyrenula nitida (Weigel) Ach. & $\mathrm{EN}$ & $57 \%$ & $36 \%$ & $42 \%$ & $22 \%$ & $8 \%$ & $67 \%$ & $\mathrm{X}$ & \\
\hline & Ramalina farinacea (L.) Ach. & EN & & $12 \%$ & & & $8 \%$ & $4 \%$ & & $\mathrm{D}$ \\
\hline & Ramalina sp. & & & & & & $4 \%$ & & & $\mathrm{~K}$ \\
\hline & Ramalina pollinaria (Westr.) Ach. & $\mathrm{VU}$ & & $4 \%$ & & & $4 \%$ & & & \\
\hline & Ropalospora viridis (Tønsberg) Tønsberg & & $5 \%$ & & & $4 \%$ & $4 \%$ & $19 \%$ & $\mathrm{X}$ & $\mathrm{D}$ \\
\hline & Scoliciosporum umbrinum (Ach.) Arnold & & & & & $9 \%$ & $8 \%$ & & & 0 \\
\hline & Strangospora moriformis (Ach.) Stein & & $5 \%$ & & & & & & & \\
\hline I & Strigula stigmatella (Ach.) R.C. Harris & $\mathrm{CR}$ & & $12 \%$ & & & $4 \%$ & & & $\mathrm{~K}$ \\
\hline $\mathrm{I}$ & Thelopsis rubella Nyl. & $\mathrm{CR}$ & $14 \%$ & & & & & $4 \%$ & & $\mathrm{H}, \mathrm{D}$ \\
\hline I & Thelotrema lepadinum (Ach.) Ach. & $\mathrm{CR}$ & & $4 \%$ & & & & $33 \%$ & $\mathrm{X}$ & \\
\hline & Trapelia corticola Coppins \& P. James & & & $4 \%$ & & & & & & $\mathrm{~S}$ \\
\hline & Trapeliopsisflexuosa (Fr.) Coppins \& P. James & & & $4 \%$ & & & & & & $S$ \\
\hline & Trapeliopsis granulosa (Hoffm.) Lumbsch & & & $4 \%$ & & & $4 \%$ & & & \\
\hline I & $\begin{array}{l}\text { Varicellaria hemisphaerica (Flörke) } \\
\text { I. Schmitt \& Lumbsch }\end{array}$ & $\mathrm{CR}$ & $5 \%$ & $4 \%$ & & $4 \%$ & & & $\mathrm{X}$ & 0 \\
\hline & Violella fucata (Stirt.) T. Sprib. & & $5 \%$ & $4 \%$ & $54 \%$ & $43 \%$ & $42 \%$ & $33 \%$ & $\mathrm{X}$ & \\
\hline & $\begin{array}{l}\text { Vulpicida pinastri (Scop.) } \\
\text { J.-E. Mattsson \& M.J. Lai }\end{array}$ & NT & & $12 \%$ & & & & & & S \\
\hline Total & 128 & 61 & 52 & 79 & 31 & 44 & 69 & 39 & 22 & \\
\hline
\end{tabular}

Tab. 7. List of all lichen species recorded on other substrates.

General abbreviations: I - indicator species of conservation value of beech forests, RL - red-listed species.

Threatened species categories: EX - extinct, CR - critically endangered, EN - endangered, VU - vulnerable, NT - near threatened, DD - data deficient.

Abbreviations of investigated sites: B - Badínsky prales, D - Dobročský prales, H - Havešová, K Klenovský Vepor, O - Oblík, S - Stužica, V - Vihorlat (for details, see Material and methods).

Species represented in herbarium SAV and their origin are indicated in the last column.

\begin{tabular}{|c|l|c|c|c|c|c|c|c|c|c|c|}
\hline Status & Species & RL & H & S & V & O & K & D & B & Substrate & SAV \\
\hline I & Arthonia spadicea Leight. & & & & & & & X & & Acer pseudoplatanus & D \\
\hline I & Arthonia vinosa Leight. & CR & & & X & & & & & Acer pseudoplatanus & V \\
\hline I & $\begin{array}{l}\text { Bryoria capillaris (Ach.) } \\
\text { Brodo \& D. Hawksw. }\end{array}$ & CR & & & & & X & & & Picea abies & $\mathrm{K}$ \\
\hline I & Collema flaccidum (Ach.) Ach. & $\mathrm{EN}$ & $\mathrm{X}$ & & & & & & & rock & $\mathrm{H}$ \\
\hline
\end{tabular}


ADAMČÍK S. ET AL.: FUNGI AND LICHENS OF NATURAL BEECH FORESTS IN SLOVAKIA

\begin{tabular}{|c|c|c|c|c|c|c|c|c|c|c|c|}
\hline Status & Species & $\mathbf{R L}$ & $\mathbf{H}$ & $\mathbf{S}$ & V & $\mathbf{0}$ & $\mathbf{K}$ & D & B & Substrate & SAV \\
\hline I & Gyalecta flotowii Körb. & $\mathrm{CR}$ & & $\mathrm{X}$ & & & & & & Abies alba & $\mathrm{S}$ \\
\hline \multirow[t]{3}{*}{ I } & Gyalecta ulmi (Sw.) Zahlbr. & CR & & & & & & $\mathrm{X}$ & & Acer platanoides & $\mathrm{D}$ \\
\hline & $\begin{array}{l}\text { Hypocenomyce caradocensis } \\
\text { (Leight. ex Nyl.) } \\
\text { P. James \& Gotth. Schneid. }\end{array}$ & & & $\mathrm{X}$ & & & & & & Abies alba & S \\
\hline & $\begin{array}{l}\text { Hypocenomyce scalaris } \\
\text { (Ach. ex Lilj.) M. Choisy }\end{array}$ & & & $\mathrm{X}$ & & & $\mathrm{X}$ & & & Abies alba & S \\
\hline \multirow[t]{5}{*}{ I } & Hypogymnia farinacea Zopf & $\mathrm{VU}$ & & & & & $\mathrm{X}$ & & & Acer pseudoplatanus & $\mathrm{K}$ \\
\hline & $\begin{array}{l}\text { Hypogymnia tubulosa (Schaer.) } \\
\text { Hav. }\end{array}$ & NT & & & & & $\mathrm{X}$ & & & Picea abies & $\mathrm{K}$ \\
\hline & $\begin{array}{l}\text { Lecanora conizaeoides } \\
\text { Nyl. ex Cromb. }\end{array}$ & & & $\mathrm{X}$ & & & & & & Abies alba & S \\
\hline & Lecanora hagenii (Ach.) Ach. & & & $\mathrm{X}$ & & & & & & Acer pseudoplatanus & $\mathrm{S}$ \\
\hline & $\begin{array}{l}\text { Lepraria membranacea (Dicks.) } \\
\text { Vain. }\end{array}$ & & & & & & $\mathrm{X}$ & & & rock & \\
\hline I & $\begin{array}{l}\text { Leptogium cyanescens (Rabenh.) } \\
\text { Körb. }\end{array}$ & $\mathrm{CR}$ & $\mathrm{X}$ & & & & & & & rock & $\mathrm{H}$ \\
\hline I & $\begin{array}{l}\text { Leptogium lichenoides (L.) } \\
\text { Zahlbr. }\end{array}$ & & $\mathrm{X}$ & & & & & & & rock & $\mathrm{H}$ \\
\hline $\mathrm{I}$ & Lobaria pulmonaria (L.) Hoffm. & $\mathrm{CR}$ & & $\mathrm{X}$ & & & & & & Acer pseudoplatanus & \\
\hline I & Nephroma parile (Ach.) Ach. & $\mathrm{CR}$ & & $\mathrm{X}$ & & & & & & Acer pseudoplatanus & \\
\hline \multirow[t]{7}{*}{ I } & $\begin{array}{l}\text { Opegrapha vermicellifera } \\
\text { (Kunze) J.R. Laundon }\end{array}$ & & $\mathrm{X}$ & & & & & & & Acer pseudoplatanus & $\mathrm{H}$ \\
\hline & Physcia adscendens H. Olivier & & & & $\mathrm{X}$ & & & & & Acer pseudoplatanus & V \\
\hline & Parmelina tiliacea (Hoffm.) Hale & NT & & & $\mathrm{X}$ & $\mathrm{X}$ & & & & Acer pseudoplatanus & $\mathrm{V}, 0$ \\
\hline & $\begin{array}{l}\text { Parmeliopsis ambigua (Wulfen) } \\
\text { Nyl. }\end{array}$ & & & $\mathrm{X}$ & & & & & & Acer pseudoplatanus & \\
\hline & $\begin{array}{l}\text { Platismatia glauca (L.) } \\
\text { W.L. Culb. \& C.F. Culb. } \\
\end{array}$ & NT & & $\mathrm{X}$ & & & & & & Acer pseudoplatanus & \\
\hline & Pseudevernia furfuracea (L.) Zopf & NT & & & & & $\mathrm{X}$ & & & Picea abies & $\mathrm{K}$ \\
\hline & Ramalina carpatica Körb. & NT & & & & & $\mathrm{X}$ & & & rock & $\mathrm{K}$ \\
\hline \multirow[t]{2}{*}{$\mathrm{I}$} & $\begin{array}{l}\text { Varicellaria hemisphaerica } \\
\text { (Flörke) I. Schmitt \& Lumbsch }\end{array}$ & $\mathrm{CR}$ & & & & $\mathrm{X}$ & & & & Acer pseudoplatanus & \\
\hline & $\begin{array}{l}\text { Vulpicida pinastri (Scop.) } \\
\text { J.-E. Mattsson \& M.J. Lai } \\
\end{array}$ & NT & & $\mathrm{X}$ & & & & & & Acer pseudoplatanus & S \\
\hline Total & 26 & 16 & 4 & 10 & 3 & 2 & 7 & 2 & $\mathbf{0}$ & & \\
\hline
\end{tabular}

\section{CONCLUSION}

In this study, Stužica showed the highest number of fungal (126) and lichen (79) species recorded on beech wood and living trees. It had the highest number (19) of fungal site specific species (collected only on single site) and the highest number (19) of lichen indicators. Including historical data, a total of 19 fungal indicators of high nature conservation value have been reported from this site, which makes it 
the first ranked among European beech forests explored so far. The second highest number of fungal species (121) and highest number of indicator species (14) recorded on beech wood was reported from Havešová. For lichens, Havešová ranks third, with 52 recorded lichens in total and 15 indicators. Klenovský Vepor is second in lichen species richness on beech trees (69) and indicators (18) and is the third richest for fungal species on beech wood (80 in total, 12 indicators). The site with the lowest number of beech wood-inhabiting species was Vihorlat (72 fungi and 31 lichens), including 8 fungal indicators. In the evaluation above, Badínsky prales is not included, because the site was only visited for a short time and just a few logs were investigated. The highest diversity at Stužica is related to the large area of continuous forest cover: it is the most isolated site distant from industrial or agricultural influence, its area is much larger than that of any of the other sites explored (the second largest reserve, Havešová, has a size of not more than a fourth), it has the largest range of expositions, the largest altitudinal range and the largest variety of habitats. Similarly, the limited diversity in Vihorlat corresponds to its small area, hilltop position directly exposed to wind and sun, low range of altitudes and the vicinity of chemical industry (in the towns of Strážske and Humenné). Both indicator systems, the fungus system by Christensen et al. (2004) and the lichen system by Pišút (1997, updated by Lackovičová \& Guttová 2005) show similar results and our research approved its reliability in assessing the conservation value of montane old-growth beech forests.

\section{ACKNOWLEDGEMENTS}

We are very grateful to two reviewers for their useful critiques and editorial improvements. Staff of the protected areas and administration units concerned are acknowledged for their help with research permits and transport to the (poorly accessible) sites: Marián Gíč (Poloniny National Park), Ladislav Palko (Vihorlat Protected Landscape Area), Anton Olah (Slovak Environment Agency), Drahoš Blanár (Muránska planina National Park) and Peter Potocký (Pol’ana Protected Landscape Area). André Aptroot (Soest, The Netherlands), Ulf Arup (University of Lund, Sweden), Zdeněk Palice (Institute of Botany of the Czech Academy of Sciences, Czech Republic) and Jiří Malíček (Charles University in Prague, Czech Republic) helped with identification of the lichens. The research conducted by Slavomír Adamčík and Soňa Jančovičová was funded from Slovak National Agency project VEGA 02/0075/14, Anna Lackovičová's work from VEGA 02/0034/13, Vladimír Kunca's work from VEGA 01/0362/13 and Jan Holec was supported by the Ministry of Culture of the Czech Republic (DKRVO 2015/08, National Museum, 00023272). The J.E. Lange foundation is thanked for financial support of the field research during the Symposium. 


\section{REFERENCES}

AdamčíK S., Christensen M., Heilmann-Clausen J., WAlleyn R. (2007): Fungal diversity in the Poloniny National Park with emphasis on indicator species of conservation value of beech forests in Europe. - Czech Mycol. 59: 67-81.

ADAMČÍK S., KUČERA V., LIZOŇ P., RIPKA J., RIPKOVÁ S. (2003): Stage of the biodiversity research on macrofungi in Slovakia. - Czech Mycol. 55: 201-213.

ANONYMUS 1 (on-line): World heritage site - for world heritage travellers. http://www.worldheritagesite.org/sites/beechforests.html. [accessed 27 July 2015]

ANONYMUS 2 (on-line): Index Fungorum. - http://www.indexfungorum.org/. [accessed 27 July 2015]

ANTONín V., Beran M., BoroviČKa J., DvořÁK D., Holec J. (2011): Clitocybula familia (Fungi, Agaricales) - taxonomy, distribution, ecology and first records in the Czech Republic and Slovakia. - Czech Mycol. 63: 1-11.

Antonín V., Fellner R., Herink J., LAzebníČEK J., Lizoň P., Kotlaba F., ŠEBeK S. (1995): Huby (makromycéty) [Fungi (macromycetes)]. - In: Kotlaba F., ed., Červená kniha ohrozených a vzácnych druhov rastlín a živočíchov SR a ČR 4 [Red book of endangered and rare species of plants and animals of Slovak Republic and Czech Republic 4], pp. 30-119, Príroda, Bratislava. [in Slovak]

APTROOT A. (1991): A monograph of the Pyrenulaceae (excluding Anthracothecium and Pyrenula) and the Requienellaceae, with notes on the Pleomassariaceae, the Trypetheliaceae and Mycomicrothelia (lichenized and non-lichenized Ascomycetes). - Biblioth. Lichenol. 44: 1-178.

ARNOLDS E.J.M., OMMERING G. VAN (1996): Bedreigde en kwetsbare paddestoelen in Nederland. Toelichting op de Rode Lijst [Endangered and vulnerable mushrooms in the Netherlands. Notes on the Red List]. - Rapport IKC Natuurbeheer 24, Wageningen. [in Dutch]

Arup U., Wilfling A., PrÜGger J., MAYrhofer H. (2001): Contributions to the lichen flora of Slovenia VIII. Lichenized and lichenicolous fungi from Veliki Sneznik. - In: McCarthy P.M., Kantvilas G., Louwhoff S.H.J.J., eds., Lichenological contributions in honour of Jack Elix, pp. 17-25, J. Cramer, Berlin, Stuttgart.

Benkert D., DÖrfelt H., HardTKe H.J., Hirsch G., Kreisel H., Krieglsteiner G.J., LÜdeRItZ M., Runge A., SChMid H., SChMitT J.A., WinterhoFF W., WÖLDECKE K., ZEHFUSS H.D., Einhellinger A., Gross G., Grosse-Brauckmann H., Nuss I., WÖLFEL G. (1992): Rote Liste der gefährdeten Großpilze in Deutschland. - Naturschutzbund Deutschland, Bonn.

BERnicCHIA A., GorJón S.P. (2010): Corticiaceae s.l. - Fungi Europaei, Vol. 12, Edizione Candusso, Alassio.

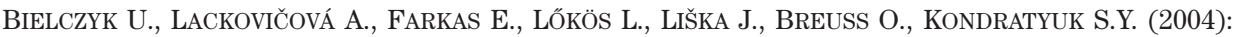
Checklist of lichens of the Western Carpathians. - W. Szafer Institute of Botany, Polish Academy of Sciences, Kraków.

Bolla J. vON (1858) [1857]: Die Pilze der Pressburger Flora. - Verh. Vereins Natur-Heilk. Pressburg 2(2): 41-71.

Christensen M., Heilmann-Clausen J., Walleyn R., ADAMČíK S. (2004): Wood-inhabiting fungi as indicators of nature value in European beech forests. - In: Marchetti M., ed., Monitoring and indicators of forest biodiversity in Europe - from ideas to operationality, EFI Proceedings 51, pp. 229-237.

DAhlberg A., Croneborg H. (2003): 33 threatened fungi in Europe, Complementary and revised information on candidates for listing in Appendix I of the Bern Convention, August 2003. http://www.artdata.slu.se/Bern_Fungi/ECCF\%2033_T-PVS\%20(2001)\%2034\%20rev_low\%20resolution_p\%201-14.pdf. [accessed 22 July 2015]

FELlneR R. (1994): Huby [Fungi]. - In: Rozložník M., Karasová E., eds., Chránená krajinná oblast' biosférická rezervácia Slovenský kras [Slovenský Kras Protected Landscape Area and Biosphere Reserve], pp. 97-102, Osveta, Martin. [in Slovak] 
GÄRDENFORS U., ed. (2010): Rödlistade arter i Sverige 2010 [The 2010 Red list of Swedish species]. ArtDatabanken, SLU, Uppsala. [in Swedish]

GLEJDURA S. (1997): Vzácne druhy húb arboréta Borová Hora vo Zvolene a rôzných častí Slovenska v rokoch 1991-1996 [Rare fungi reported from Borová hora arboretum in Zvolen and various parts of Slovakia in 1991-1996]. - Sprav. Slov. Mykol. 18: 24-28. [in Slovak]

GLEJDURA S. (2002): Zoznam húb [The list of fungi]. - In: Slávik D., ed., Dobročský prales - Národná prírodná rezervácia [Dobročský prales - National Nature Reserve], pp. 48-55, Zvolen. [in Slovak]

GLEJDURA S. (2013): Nové nálezy bazídiových a vreckatých húb v Stolických vrchoch (Slovensko) [New records of basidiomycetes and ascomycetes in the Stolické vrchy Mts. (Slovakia)]. - Mykol. Listy 124: 15-40. [in Slovak]

Groner U. (2006): The genus Chaenothecopsis (Mycocaliciaceae) in Switzerland, and a key to the European species. - Lichenologist 38: 395-406.

GuTTOVÁ A. (1997): Príspevok k poznaniu lichenizovaných ascomycét slovenskej časti Východných Karpát [Contribution to the distribution of lichenized ascomycetes in the Eastern Carpathians on the territory of Slovakia]. - Bull. Slov. Bot. Spoločn. 19: 78-83. [in Slovak]

GUTTOVÁ A., LACKOVIČOvÁ A. (2011): Pralesy a lišajníky [Old-growth forests and lichens]. - In: Jasík M., Polák P., eds., Pralesy Slovenska [Old-growth forests of Slovakia], pp. 31-34, Banská Bystrica. [in Slovak]

GuTtová A., LACKOVIČová A., PIŠÚt I. (2013): Revised and updated checklist of lichens of Slovakia (May 2013). - Biologia, Bratislava 68: 845-850 (electron. incl. Appendix: 1-50).

GuTtová A., PALICE Z. (1999): Lišajníky Národného parku Muránska planina I - Hrdzavá dolina [Lichens of Muránska planina National Park I - Hrdzavá dolina valley]. - In: Uhrin M., ed., Výskum a ochrana prírody Muránskej planiny 2 [Research and nature conservation of Muránska planina 2], pp. 35-47, Revúca. [in Slovak]

GuTTOVÁ A., PALICE Z. (2001): Výskyt jamkatca plúcneho (Lobaria pulmonaria) v NP Muránska planina (Stredné Slovensko) [Occurrence of Lobaria pulmonaria in Muránska planina National Park (Central Slovakia)]. - Bull. Slov. Bot. Spoločn. 23: 39-43. [in Slovak]

GuTTOVÁ A., PALICE Z. (2002): Lišajníky Národného parku Muránska planina II - Javorníková dolina [Lichens of Muránska planina National Park II - Javorníková dolina valley]. - In: Uhrin M., ed., Výskum a ochrana prírody Muránskej planiny 3 [Research and nature conservation of Muránska planina 3], pp. 53-68, Revúca. [in Slovak]

GUTTOVÁ A., PALICE Z. (2004): Lišajníky Národného parku Muránska planina III - Cigánka [Lichens of Muránska planina National Park III - Cigánka]. - Reussia 1, Suppl. 1: 5-40.

GUTTOVÁ A., PIŠÚT I. (2004): Composition of lichen-diversity in the biocentre Strážovské vrchy Mts. recent and unpublished data. - In: Franc V., ed., Strážovské vrchy Mts. - research and conservation of nature, pp. 5-16, Belušské Slatiny.

HAGARA L. (1989): Vzácne prvky slovenskej mykoflóry [Rare elements of the Slovak mycota]. - Česká Mykol. 43: 51-60. [in Slovak]

HAGARA L. (2001): Distribution of corticioid fungi in Slovakia: Botryobasidium and related genera. Catathelasma 1: 8-21.

HAGARA L. (2004): Hyphodontia in Slovakia: distribution and ecology. - Catathelasma 5: 3-21.

HAGARA L. (2014) [2015]: Ottova encyklopédia húb [Otto's encyclopaedia of fungi]. - Ottovo nakladatelství, Praha.

HANSEn L., KNUDSEn H., eds. (2000): Nordic Macromycetes, Vol. 1: Ascomycetes. - Nordsvamp, Copenhagen.

HANSEn L., KnUdSen H., CoRfiXen P., eds. (1997): Nordic Macromycetes, Vol. 3: Heterobasidioid, Aphyllophoroid and Gastromycetoid Basidiomycetes. - Nordsvamp, Copenhagen.

HAZSLINSZKY F. (1862): Eperjes viránya zuzmói [Lichens from Prešov region]. - Math. Term. Közlem. 2: 162-230. [in Hungarian]

HAZSLINSZKY F. (1884): A Magyar birodalom zuzmó-flórája [Lichen flora of the Hungarian kingdom]. Kir. Magyar Term. Társ. Évk., Budapest. [in Hungarian] 
HEILMANN-ClAUSEN J. (2001): A gradient analysis of communities of macrofungi and slime moulds on decaying beech logs. - Mycol. Res. 105: 575-596.

HOLEC J. (2005): Distribution and ecology of Camarops tubulina (Ascomycetes, Boliniaceae) in the Czech Republic and remarks on its European distribution. - Czech Mycol. 57: 97-115.

HoLEC J. (2012): Tricholomopsis osiliensis (Basidiomycota, Agaricales), recently described from Estonia, found in Slovakia. - Czech Mycol. 64: 93-100.

HOLEC J., BERAN M., eds. (2006): Červený seznam hub (makromycetů) České republiky [Red list of fungi (macromycetes) of the Czech Republic]. - Příroda 24: 1-282. [in Czech]

HOLEC J., KOLAŘíK M. (2013): Ossicaulis lachnopus (Agaricales, Lyophyllaceae), a species similar to O. lignatilis, is verified by morphological and molecular methods. - Mycol. Progr. 12: 589-597.

JANČOVIČOvÁ S., ADAMČÍK S. (2012): Entoloma jahnii (Fungi, Agaricales) reported from Slovakia and notes on differences with E. byssisedum. - Czech Mycol. 64: 209-222.

JANČOVIČOVÁ S., TOMŠOVSKÝ M., URBAN A., KRISAI-GREILHUBER I. (2014): New records and epitypification of Crepidotus malachioides (Crepidotaceae, Agaricales) - Sydowia 66: 79-97.

JEANnERot B. (2011): Contribution à l'étude du genre Scutellinia (Pezizales). I: Scutellinia decipiens Le Gal. - Bull. Mycol. Bot. Dauphiné-Savoie 200: 51-64.

Kabát V., Tomáñ P., Beran M., Deckerová H., Janitor A., Milan I., Gle.Jdura S., VaŠUtová M. (2007): Výskyt makromycétov v okolí Malatinej [Occurrence of macromycetes in the vicinity of Malatiná]. - Zborník Oravského múzea 24: 105-127. [in Slovak]

Khodosovtsev O., Dymytrova L., Nadyeina O., Naumovych A., Khodosovtseva Y., ScheidegGer C. (2013): A contribution to beech forest-associated epiphytic lichen-forming and lichenicolous fungi in Crimean Mts (Ukraine). - Fl. Medit. 23: 57-68.

KNUDSEN H., VESTERHOLT J., eds. (2012): Funga Nordica. Agaricoid, boletoid and cyphelloid genera. Nordsvamp, Copenhagen.

KoTLABA F. (1984): Zeměpisné rozšíření a ekologie chorošů (Polyporales s. 1.) v Československu [Geographical distribution and ecology of polypores (Polyporales s. 1.) in Czechoslovakia]. Academia, Praha. [in Czech]

KoTLABA F. (2000): Třetí příspěvek k houbám Soběslavských blat [Third supplement to the fungi of the "Soběslavská blata" peat bogs]. - Mykol. Listy 73: 1-13. [in Czech]

KoTlaba F., PouZar Z. (1962): Lupenaté a hřibovité houby (Agaricales) Dobročského pralesa na Slovensku [Agarics and boletes (Agaricales) of Dobročský prales in Slovakia]. - Česká Mykol. 16: 173-191. [in Czech]

Kotlaba F., PouZAr Z., SvrčEk M. (1991): Huby [Fungi]. - In: Vološčuk I., Pelikán V., eds., Chránená krajinná oblast' Muránska planina [Muránska planina Protected Landscape Area], pp. 76-82, Bratislava. [in Slovak]

KRIŽOvÁ E. (2002): Vegetácia [Vegetation]. - In: Slávik D., ed., Dobročský prales - Národná prírodná rezervácia [Dobročský prales - National Nature Resrve], pp. 18-24, Zvolen. [in Slovak]

KulT K. (1989): Súpis druhov húb zbieraných na exkurziách 29. IX.-3. X. 1986 v okolí Spišskej Novej Vsi a na d’alších lokalitách účastníkmi 4. mykologických dní na Slovensku [List of species collected during the excursions from Sept. 29 to Oct. 3, 1986 near Spišská Nová Ves and other sites by participants of the $4^{\text {th }}$ mycological meeting in Slovakia]. - In: Kuthan J., ed., Houby rašeliništ a bažinatých lesů v Československu [Fungi of peat-bogs and marshy forests in Czechoslovakia], pp. 42-62, Praha. [in Slovak]

KULT K. (1991): Soupis druhů hub sbíraných při mykofloristické akci sekce pro mykofloristiku a mykocenologii ČSVSM ve Slanských vrchoch [List of species collected during the mycological meeting of the section for fungal diversity and fungal coenology ČSVSM in the Slanské vrchy Mts.]. - In: Kuthan J., ed., Houby rostoucí v prostředí ovlivněném činností člověka [Fungi growing in environments with human impact], pp. D1-D22, Ostrava. [in Czech]

KUTHAN J. (1984): Seznam makromycetů sbíraných účastníky 3. mykologických dnů na Slovensku ve Skýcově v průběhu exkursí a pobytu ve dnech 4.-9. 10. 1983 [List of macrofungi collected by participants of the $3^{\text {rd }}$ mycological meeting in Skýcov, Slovakia during excursions and stay from Oct 
4 to 9, 1983]. - In: Kuthan J., ed., Houby teplomilných doubrav Československa [Fungi of thermophilous oak forests in Czechoslovakia], pp. 30-35, Praha. [in Czech]

Kuthan J., AdAmČíK S., ANTONín V., TERRAY J. (1999): Huby Národného parku Poloniny [Fungi of Poloniny National Park]. - Správa národných parkov SR, Liptovský Mikuláš, Správa Národného parku Poloniny, Snina, Košice. [in Slovak]

KUTHAN J., SINGER R. (1987): Mykoflóra horské smrčiny v oblasti Štrbského plesa (Vysoké Tatry) [Fungal diversity of montane spruce forests in the area of Štrbské pleso (High Tatra Mts.)]. - In: Kuthan J., ed., Houby horských smrčin a podhorských smrkových porostů v Československu [Fungi of montane and sub-montane spruce forests in Czechoslovakia], pp. 27-33, Praha. [in Czech]

LACKOVIČOvÁ A., GuTTOvÁ A. (2005): Genus Dimerella (Coenogoniaceae, lichenized Ascomycota) in Slovakia. - Bot. Croatica 64: 289-301.

LACKOVIČOvÁ A., GUTTOVÁ A. (2006): Lichen diversity - history, contemporary occurrence and trend in Slovakia: Gyalecta ulmi and Leptogium saturninum. - In: Lackovičová A., Guttová A., Lisická E., Lizoň P., eds., Central European lichens - diversity and threat, pp. 219-240, Ithaca.

LACKOviČOvÁ A., GuTTOVÁ A., PIŠÚT I. (2004): Diverzita lišajníkov Národnej prírodnej rezervácie Vihorlatský prales (Vihorlat, východné Slovensko) [Lichen diversity of Vihorlatský prales National Nature Reserve (Vihorlat Mts., East Slovakia)]. - Bull. Slov. Bot. Spoločn., Suppl. 10: 96-100. [in Slovak]

LACKOVIČOVÁ A., LISICKÁ E., LISICKÝ M.J., GUTTOVÁ A. (2001): Contribution to conservation of lichens throughout Europe. - Bryonora 27: 2-9.

LACKOVIČOvÁ A., PIŠÚT I. (2004): Lišajníky NPR Dobročský prales (Klenovský Vepor, Veporské vrchy) [Lichens of Dobročský prales National Nature Reserve (Klenovský Vepor, Veporské vrchy Mts.)]. - Bull. Slov. Bot. Spoločn. 26: 23-29.

LISICKÁ E. (2005): The lichens of the Tatry Mountains. - Veda, Bratislava.

LISICKÁ E. (2006): Diversity of epiphytic lichens of the Tatry Mountains, Slovakia. - In: Lackovičová A., Guttová A., Lisická E., Lizoň P., eds., Central European lichens - diversity and threat, pp. 283-304, Ithaca.

LizoŇ P. (2001): Červený zoznam húb Slovenska, 3. verzia (december 2001) [Red list of fungi of Slovakia, third draft (December 2001)]. - In: Baláž D., Marhold K., Urban P., eds., Červený zoznam rastlín a živočíchov Slovenska [Red list of plants and animals of Slovakia], Ochr. Prír. 20 (Suppl.): 6-13. [in Slovak]

LUKNIŠ M., MAZÚR E. (1980): IV. Povrch, 16. Geomorfologické jednotky [IV. Surface, 16. Geomorphological units]. - In: Mazúr E., Jakal J., eds., Atlas Slovenskej socialistickej republiky [Atlas of the Slovak Socialistic Republic], pp. 54-55, Slovenská akadémia vied, Slovenský úrad geodézie a kartografie, Bratislava. [in Slovak]

MALíčEK J., PALICE Z., VONDRÁK J. (2014): New lichen records and rediscoveries from the Czech Republic and Slovakia. - Herzogia 27: 257-284.

MietTinen O., Niemelä T., SPIRIN W. (2006): Northern Antrodiella species: the identity of A. semisupina, and type studies of related taxa. - Mycotaxon 96: 211-239.

MiHÁL I. (1996): Mykoflóra podhorskej jedlovej bučiny v Kremnických vrchoch [Fungal diversity of sub-montane fir-beech forest in the Kremnické vrchy Mts.]. - Sprav. Slov. Mykol. 13: 21-23. [in Slovak]

MiHÁL I., BlanáR D. (2011): Huby rodov Hypocrea s.l., Hypomyces s.l. a Nectria s.l. (Hypocreaceae, Bionectriaceae, Nectriaceae, Ascomycota) zistené v oblasti horného a stredného Gemera [Fungi of genera Hypocrea s.l., Hypomyces s.l. and Nectria s.l. (Hypocreaceae, Bionectriaceae, Nectriaceae, Ascomycota) recorded in the area of upper and central Gemer region]. - Reussia 6: 45-86. [in Slovak]

Mihál I., Cicák A., TSAKov H., PetKov P. (2009): Occurrence of species of the Nectria s.l. (Bionectriaceae, Nectriaceae, Hypocreales, Ascomycetes) in Central and South-eastern Europe. Folia Oecol. 36: 32-38. 
NiKLFELD H. (1971): Bericht über die Kartierung der Flora Mitteleuropas. - Taxon 20: 545-571.

Nimis P.L. (1993): The lichens of Italy. An annotated catalogue. - Museo Regionale di Scienze Naturali, Torino.

Ódor P., Heilmann-Clausen J., Christensen M., Aude E., Dort K.W. van, Piltaver A., Siller I., Veerkamp M.T., WAlleyn R., Standovár T., HeEs A.F.M. van, Kosec J., MAToČEC N., Kraigher H., GREBENC T. (2006): Diversity of dead wood inhabiting fungi and bryophytes in semi-natural beech forests in Europe. - Biol. Conservation 131: 58-71.

PALICE Z. (1999): New and noteworthy records of lichens in the Czech Republic. - Preslia 71: 289-336.

PILÁT A. (1926): Les Agaricales et Aphyllophorales des Carpathes Centrales. - Bull. Soc. Mycol. France 42: 81-120.

PIŠÚT I. (1963): Príspevok k poznaniu lišajníkov Slovenska III [Contribution to the knowledge of lichens of Slovakia III]. - Acta Fac. Rerum Nat. Univ. Comenianae, Bot. 8: 359-364. [in Slovak]

PIŠÚT I. (1985): Die aktuelle Verbreitung einiger epiphytischen Flechtenarten in der Slowakei I. Zborn. Slov. Nár. Múz., Prír. Vedy 31: 3-26.

PIŠÚT I. (1986): Nachträge zur Kenntnis der Flechten der Slowakei II. - Zborn. Slov. Nár. Múz., Prír. Vedy 32: 167-170.

PIŠÚT I. (1987): Lišajníky [Lichens]. - In: Vološčuk I., Terray J., eds., Chránená krajinná oblast' Vihorlat [Vihorlat Protected Landscape Area], pp. 61-65, Bratislava.

PIŠÚT I. (1997): Application of some epiphytic lichens for environmental valorisation of mountain forests in Slovakia. - Biologia, Bratislava 52: 23-26.

PIŠÚT I. (1999): Mapovanie rozšírenia epifytických lišajníkov na Slovensku (1970-1981) [Mapping of the distribution of epiphytic lichens in Slovakia (1970-1981)]. - Botanický ústav SAV, Bratislava. [in Slovak]

PIŠÚT I. (2000): Nachträge zur Kenntnis der Flechten der Slowakei 14. - Acta Rer. Natur. Mus. Slov. 46: 11-14.

PIŠÚT I., GuTTOVÁ A. (1997): A few rare or overlooked lichenized ascomycetes from Slovakia. Biologia, Bratislava 52: 495-498.

PIŠÚT I., GuTTOVÁ A., LACKOviČOvÁ A., LisickÁ E. (2001): Červený zoznam lišajníkov Slovenska (december 2001) [Red list of lichens of Slovakia (December 2001)]. - In: Baláž D., Marhold K., Urban P., eds., Červený zoznam rastlín a živočíchov Slovenska [Red list of plants and animals of Slovakia], Ochr. Prír. 20 (Suppl.): 23-30. [in Slovak]

PIŠÚT I., LACKOVIČOVÁ A. (1992): Flechten der Staatlichen Natur-Reservation Stužica (Gebirge Bukovské vrchy, Ostslowakei). - Biologia, Bratislava 47: 549-559.

PIŠÚT I., LACKOvičovÁ A., GUTTOVÁ A., PALICE Z. (2007): New lichen records from Bukovské vrchy Mts (NE Slovakia). - Acta Mycol. 42: 267-280.

PIŠÚT I., LIŠKA J. (1985): Lišajníky Slanských vrchov [Lichens of the Slanské vrchy Mts.]. - Zborn. Slov. Nár. Múz., Prír. Vedy 31: 27-57.

PIŠÚT I., LIŠKA J. (1995): Lišajníky [Lichens]. - In Kotlaba F., ed., Červená kniha ohrozených a vzácnych druhov rastlín a živočíchov SR a ČR 4 [Red book of endangered and rare plant and animal species of the Slovak Republic and Czech Republic 4], pp. 120-156, Príroda, Bratislava. [in Slovak]

POUZAR Z. (1987): Hypoxylon macrocarpum Pouz. spec. nov., a new fragrant pyrenomycete. - Česká Mykol. 32: 19-21.

RiMócZi I., Siller I., VASsAs G., AlBeRT L., VeTTER J., BRATEK Z. (1999): Magyarország nagygombáinak javasolt Vörös Listája [Red list of macrofungi of Hungary]. - Clusiana 38: 107-132. [in Hungarian].

RIPKOVÁ S., ADAMČÍK S., KUČERA V., PALKO L. (2007): Fungi of the Protected Landscape Area Vihorlat/ Huby Chránenej krajinnej oblasti Vihorlat. - Institute of Botany of the Slovak Academy of Sciences, Bratislava. [in English and Slovak]

SCHUMACHER T. (1990): The genus Scutellinia (Pyronemataceae). - Opera Bot. 101: 5-105. 
SERvíT M., NÁDVORNíK J. (1932): Flechten aus der Čechoslovakei II. Karpatorussland und Südostslovakei. - Věst. Král. České Společn. Nauk, Třr. Mat.-Přír. 1931: 1-42.

ŠKUBLA P. (1996): Vzácnejšie nálezy roku 1995 [Rare records of the year 1995]. - Sprav. Slov. Mykol. 11: 28-29. [in Slovak]

ŠKUBLA P. (1998a): Vzácnejšie nálezy roku 1997 [Rare records of the year 1997]. - Sprav. Slov. Mykol. 19: 25-29. [in Slovak]

ŠKUBLA P. (1998b): Príspevok k mykoflóre Belianskych Tatier [Contribution to the mycoflora of the Belianske Tatry Mts.]. - Sprav. Slov. Mykol. 21-22: 51-55. [in Slovak]

STOLTzE M., PiHL S. (1998): Rødliste 1997 over Planter og Dyr i Danmark [Red list of plant and animal species of Denmark]. - Danmarks Miljøundersøgelser og Skov- og Naturstyrelsen, Copenhagen. [in Danish]

Suija A., Czarnota P., Himelbrant D., Kowalewska A., Kukwa M., Kuznetsova E., Leppik E., Motiejūnaité J., Piterāns A., Schiefelbein U., Skazina M., Sohrabi M., Stepanchikova I., VERES K. (2010): The lichen biota of three nature reserves in island Saaremaa, Estonia. - Folia Cryptog. Estonica 47: 85-96.

SUZA J. (1923): Lichenes Slovakiae (Ad distributionem geographicam adnotationum pars prima). Acta Bot. Bohem. 2: 25-39.

SvRČEK M. (1960): Tomentelloideae Czechoslovakiae. Genere resupinata familiae Thelephoraceae s. str. - Sydowia 14: 170-245.

SvRČEK M. (1981): List of Operculate Discomycetes (Pezizales) recorded from Czechoslovakia II. (O-W). - Česká Mykol. 35: 64-89.

SVRČEK M. (1987): Mykoflóra horských smrčin „Doliny siedmich prameňov“ v Belianských Tatrách [Fungal diversity of montane spruce forests in the „Dolina siedmich prameňov“ valley, Belianske Tatry Mts.]. - In: Kuthan J., ed., Houby horských smrčin a smrkových porostů v Československu [Fungi of montane and sub-montane spruce forests in Czechoslovakia], pp. 38-40, Praha.

SzATALA Ö. (1916): Adatok Ung vármegye zuzmóflórájának ismeretéhez [Contribution to the knowledge of lichen diversity in the Ungvár county]. - Bot. Közlem. 15: 17-50. [in Hungarian]

SzATALA Ö. (1923) [1922]: Újabb adatok Ungmegye zuzmóflorájának ismeretéhez [New contribution to the lichen flora of Ung]. - Magyar Bot. Lapok 21: 33-63.

THIERS B. (on-line): Index Herbariorum: A global directory of public herbaria and associated staff. http://sweetgum.nybg.org/ih/. [accessed 22 July 2015]

TOMÁŇ P., KABÁT V. (2004): Vzácne druhy makromycétov oravskej prírody [Rare species of macrofungi of nature in Orava]. - Zborník Oravského múzea 21: 178-184. [in Slovak]

URBANAVICHYUS G. (2010): A checklist of the lichen flora of Russia. - Nauka, St. Petersburg.

VARJÚ L. (1994): Zoznam trúdnikotvarých húb nájdených v Dobročskom pralese 9.-10. októbra 1993 [List of polypores collected in Dobročský prales Oct. 9 to 10, 1993]. - Sprav. Slov. Mykol. 2: 38. [in Slovak]

VAŠUtOvÁ M. (2006): Preliminary checklist of the genus Psathyrella in the Czech Republic and Slovakia. - Czech Mycol. 58: 1-29.

VONDRÁK J., MALÍČEK J., ŠOuN J., POUSKA V. (2015): Epiphytic lichens of Stužica (E Slovakia) in the context of Central European old-growth forests. - Herzogia 28: 108-130.

WIELE N. VAN DE, STALPERS J., eds. (on-line): Mycobank database, fungal databases, nomenclature \& species banks. - http://www.mycobank.org/. [accessed 27 July 2015]

WiRTH V., HAUCK M., SCHUlTZ M. (2013): Die Flechten Deutschlands. - Ulmer, Stuttgart.

WOJEWODA W., ŁAWRYNOWICZ M. (1992): Red list of threatened macrofungi in Poland. - In: Zarzycki K., Wojewoda W., Heinrich Z., eds., List of threatened plants in Poland, $2^{\text {nd }}$ ed., pp. 27-56, Polish Academy of Sciences, Kraków.

ZAHLBRUCKNER A. (1894) [1892/93]: Zur Flechtenflora des Pressburger Komitates. - Verh. Vereins Natur-Heilk., Pressburg, Neue Folge 8: 19-73, 273-284. 